\title{
Distinct colonization patterns and CDNA-AFLP transcriptome profiles in compatible and incompatible interactions between melon and different races of Fusarium oxysporum f. sp. melonis
}

Sara Sestili ${ }^{1}$, Annalisa Polverari ${ }^{2 *}$, Laura Luongo ${ }^{3}$, Alberto Ferrarini ${ }^{2}$, Michele Scotton $^{4}$, Jamshaid Hussain ${ }^{2}$, Massimo Delledonne ${ }^{2}$, Nadia Ficcadenti ${ }^{1}$, Alessandra Belisario ${ }^{3^{*}}$

\begin{abstract}
Background: Fusarium oxysporum f. sp. melonis Snyd. \& Hans. (FOM) causes Fusarium wilt, the most important infectious disease of melon (Cucumis melo L.). The four known races of this pathogen can be distinguished only by infection on appropriate cultivars. No molecular tools are available that can discriminate among the races, and the molecular basis of compatibility and disease progression are poorly understood. Resistance to races 1 and 2 is controlled by a single dominant gene, whereas only partial polygenic resistance to race 1,2 has been described. We carried out a large-scale cDNA-AFLP analysis to identify host genes potentially related to resistance and susceptibility as well as fungal genes associated with the infection process. At the same time, a systematic reisolation procedure on infected stems allowed us to monitor fungal colonization in compatible and incompatible host-pathogen combinations.
\end{abstract}

Results: Melon plants (cv. Charentais Fom-2), which are susceptible to race 1,2 and resistant to race 1, were artificially infected with a race 1 strain of FOM or one of two race 1,2 w strains. Host colonization of stems was assessed at 1, 2, 4, 8, 14, 16, 18 and 21 days post inoculation (dpi), and the fungus was reisolated from infected plants. Markedly different colonization patterns were observed in compatible and incompatible host-pathogen combinations. Five time points from the symptomless early stage (2 dpi) to obvious wilting symptoms ( $21 \mathrm{dpi}$ ) were considered for CDNA-AFLP analysis. After successful sequencing of 627 transcript-derived fragments (TDFs) differentially expressed in infected plants, homology searching retrieved 305 melon transcripts, 195 FOM transcripts expressed in planta and 127 orphan TDFs. RNA samples from FOM colonies of the three strains grown in vitro were also included in the analysis to facilitate the detection of in planta-specific transcripts and to identify TDFs differentially expressed among races/strains.

Conclusion: Our data suggest that resistance against FOM in melon involves only limited transcriptional changes, and that wilting symptoms could derive, at least partially, from an active plant response.

We discuss the pathogen-derived transcripts expressed in planta during the infection process and potentially related to virulence functions, as well as transcripts that are differentially expressed between the two FOM races grown in vitro. These transcripts provide candidate sequences that can be further tested for their ability to distinguish between races.

Sequence data from this article have been deposited in GenBank, Accession Numbers: HO867279-HO867981.

\footnotetext{
* Correspondence: annalisa.polverari@univr.it; alessandra.belisario@entecra.it

${ }^{2}$ Department of Biotechnology, University of Verona, Strada Le Grazie 15,

37134 Verona, Italy

${ }^{3}$ Agricultural Research Council (CRA), Plant Pathology Research Center, Via C.

G. Bertero 22, 00156 Roma, Italy

Full list of author information is available at the end of the article
} 


\section{Background}

Fusarium oxysporum Schltdl.:Fr. is an anamorphic fungal soil-borne facultative parasite present in soil and on organic substrates worldwide. The species includes nonpathogenic and pathogenic strains, the latter causing vascular wilt and root rot on many economically important crops. Pathogenic F. oxysporum strains have been subdivided into over 70 different host-specific forms (formae speciales or $\mathrm{f}$. sp.) which are morphologically indistinguishable and represent intra-specific groups of strains with similar or identical host range [1,2]. The identification of pathogenic F. oxysporum isolates is traditionally based on pathogenicity testing, which is time consuming and laborious. A forma specialis can be further subdivided into races on the basis of characteristic virulence patterns on differential host cultivars [3].

Among the eight formae speciales that attack cucurbits, only F. oxysporum f. sp. melonis Snyder \& Hans. (FOM) is specific to melon (Cucumis melo L.) and it is responsible for the most important infectious disease in this fruit species [4]. Four races of the pathogen $(0,1,2$ and 1,2) have been defined according to the host resistance genes overcome by variants of the pathogen [5]. Race 1,2 is further subdivided into race 1,2 y, which causes yellowing, and race $1,2 \mathrm{w}$, which causes wilting. Race 0 induces disease on melon genotypes that lack FOM resistance genes. Two dominant, independently-inherited resistance (R) genes (Fom-1 and Fom-2) provide resistance to races 0 and 2 , and races 0 and 1 , respectively [5]. The presence of both genes confers high resistance to races 0,1 , and 2 [6]. Another gene, Fom-3, has been reported to confer resistance to races 0 and 2 in cultivar Perlita FR, but there are conflicting data suggesting allelism with Fom-1 [7]. Resistance to race 1,2 is complex and appears to be controlled by multiple recessive genes. Partial resistance was found in several Far-Eastern lines such as Ogon 9, and was introgressed into the cultivar 'Isabelle' [8] from which the two doubled-haploid resistant lines Nad-1 and Nad-2 were derived [9]. Perchepied and Pitrat [8] estimated that 4-14 genes were involved in resistance against FOM race 1,2, confirming its polygenic nature. QTL analysis revealed nine loci linked to this trait in melon [10]. More recently, Herman and Perl-Treves [11] found that two complementary recessive genes in the genotype BIZ are required to confer full resistance to race 1,2. Furthermore, a major recessive QTL for resistance was located and linked to a locus controlling fruit netting [12].

Wilting symptoms and plant death caused by FOM (particularly by race 1,2 ) can be devastating, with losses as high as $100 \%[13,9,14]$. Once introduced into the field, FOM can persist even after rotation with non-host crops, due to the production of chlamydospores (resting and durable spores) and its ability to colonize crop residues and roots of most crops grown in rotation [15]. Effective control can be achieved only through host resistance. Although many Fusarium species can penetrate into the cortical tissue of roots, only host-specific strains can penetrate the vascular elements by mycelial growth and the formation of microconidia, transported in the sap stream [2]. Unfortunately, molecular discrimination of $F$. oxysporum isolates is seriously complicated by the polyphyletic nature of many formae speciales, and isolates belonging to different formae speciales may be more related than isolates belonging to the same forma specialis [16]. Ideally, it would be possible to distinguish F. oxysporum strains based on DNA sequences directly related to (host-specific) pathogenicity or nonpathogenicity [16].

Penetration of host roots is an active process, although it may be accelerated by wounding. The progress of the infection for xylem-colonizing $F$. oxysporum strains has been documented in studies using green fluorescent protein (GFP) as a marker, mainly in melon $[17,2,18,19,12,20]$ but also in Arabidopsis and tomato [21,22]. Wilting is the outcome of a combination of regulated host-pathogen activities beginning with recognition of the host root, followed by differentiation and attachment of an appressorium-like structure, penetration of root cortex to access the vascular tissue, adaptation to the hostile plant environment, hyphal proliferation and production of microconidia within the xylem vessels, and finally the secretion of small molecules such as peptides or toxins [18,2]. The host responds with molecular defenses and with the production of defence structures including gels, gums, and tyloses, and vessels crashing by proliferation of adjacent parenchyma cells [23,24]. Understanding the molecular aspects of the infection process could shed light on the mechanisms and genes involved in the signal cascades associated with resistance and susceptibility. The response to F. oxysporum, as a vascular pathogen, has predominantly been characterized in the host/pathogen binomial tomato/ F. oxysporum f. sp. lycopersici which has become a model system for the molecular basis of disease resistance and susceptibility [25]. Some resistance mechanisms have been determined by gene silencing or insertional mutagenesis $[18,2,26,27]$. Understanding susceptibility/resistance in melon would facilitate the development of new control strategies and the identification of pathogen and host factors required for resistance responses and/or disease progression.

Changes in host and pathogen steady state mRNA levels during a fungal infection can provide a valuable readout of the molecular processes underlying resistance and susceptibility [28]. DNA microarrays are traditionally the standard tool for genome-wide expression analysis, although next-generation sequencing technologies 
are emerging as a robust alternative, but in both cases large collections of known transcript sequences must already be available [29]. In contrast, cDNA-AFLP remains the method of choice where the focus is gene discovery, particularly when dealing with plant-microbe interactions and seeking to identify transcripts from both interacting partners [30,31].

Here we describe the identification of differentially expressed transcripts in the binomial interaction between melon and FOM. The cultivar Charentais Fom2 was chosen as the host genotype since it is susceptible to FOM race 1,2 but resistant to race 1, thus providing the opportunity to investigate both compatible and an incompatible interactions in the same genetic background. We infected plants with FOM strain ISPaVe1070 (race 1) and strains ISPaVe1018 and ISPaVe1083 (race 1,2 w). The race 1,2 w strains are both highly virulent, but only ISPaVe1083 commonly induces necrosis at the collar level. These strains were chosen to identify possible differences in gene expression between isolates differing in their aggressiveness. Host colonization in stems was assessed at 1, 2, 4, 8, 14, 16,18 and 21 days post inoculation (dpi), and the fungal strains were reisolated from infected plants. We observed markedly different colonization patterns when comparing compatible and incompatible host-pathogen combinations. Five time points $(0,2,4,8$ and 21 days) from the symptomless early stage $(2 \mathrm{dpi})$ to obvious wilting symptoms (21 dpi) were considered for cDNAAFLP analysis to identify both early signaling events occurring in the plant, and plant or fungal genes possibly involved in symptom development. Because of the increase in fungal mass at late time points, the analysis was expected to identify a large number of fungal transcripts expressed in planta, particularly at $21 \mathrm{dpi}$, when wilting symptoms in the compatible interaction are obvious. RNA from colonies of the three strains grown in vitro was also included in the analysis to help detect FOM transcripts specifically expressed in planta and to identify transcript-derived fragments (TDFs) that are differentially expressed among races/strains.

\section{Results}

Melon colonization by Fusarium oxysporum f. sp. melonis races 1 and 1,2

The frequency of reisolations along the stem of inoculated melon plants at eight time points $(1,2,4,8,14$, 16,18 and $21 \mathrm{dpi}$ ) is shown for the three strains of FOM in Figure 1. Both race 1 and race 1,2 were recovered from the stems of inoculated plants, irrespective of the compatibility of the host-pathogen combination, but the strains differed in the speed and extent of colonization. Avirulent strain ISPaVe1070 (race 1) achieved a more rapid and continuous colonization of the stem
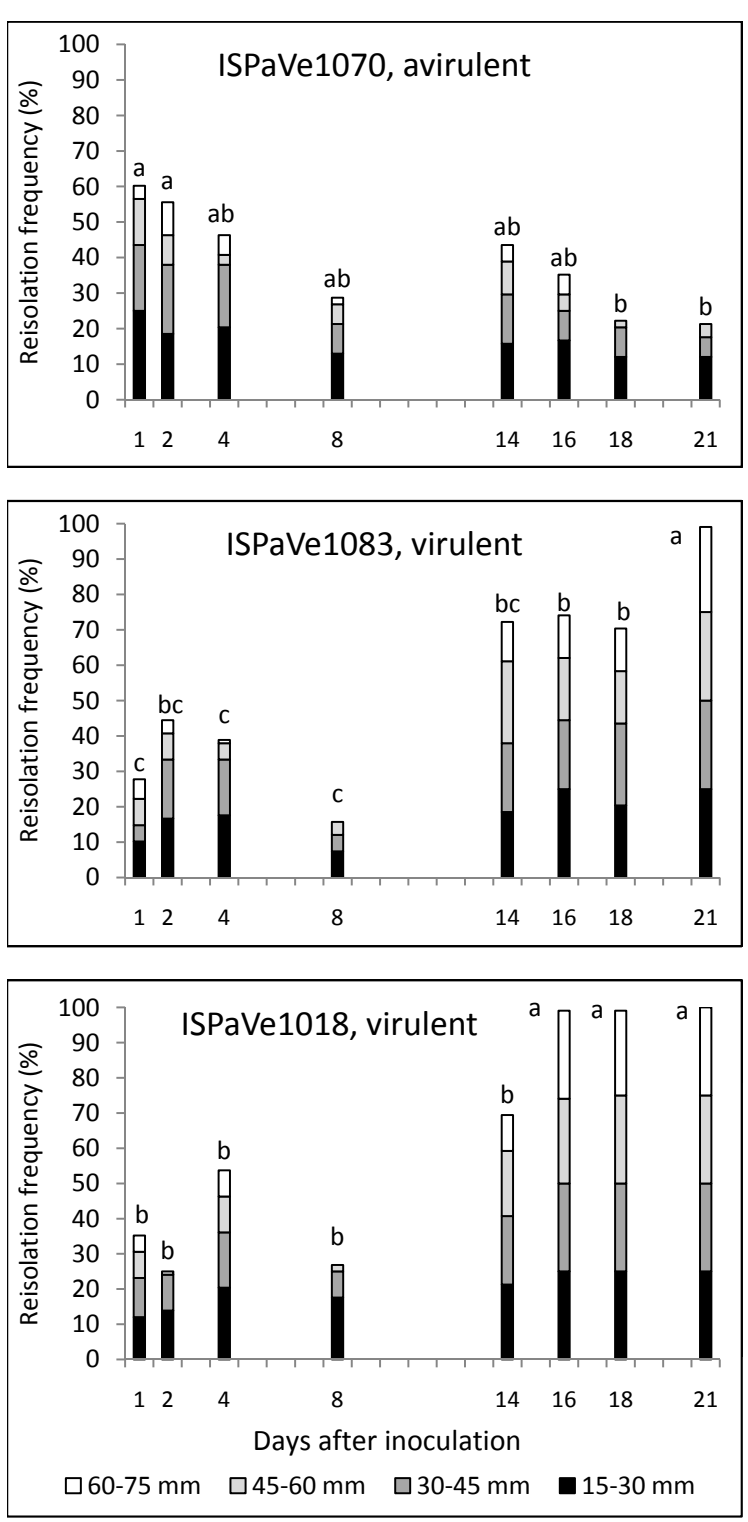

Figure 1 Vascular colonization patterns of Fusarium oxysporum f. sp. melonis (ISPaVe1070 race1, and ISPaVe1018 and 1083 race 1,2 w) on Charentais Fom-2 within a period of 21 days after inoculation. The frequency of reisolation (\%) is based on fungal colonies obtained from fragments $5 \mathrm{~mm}$ in length, cut along the stem to a maximum height of $75 \mathrm{~mm}$. The values of the four sections of each column were calculated by summing the numbers of reisolations for each of the four height classes (15-30 mm, 30-45 mm, 45-60 $\mathrm{mm}$ and 60-75 mm). Each section of the column shows the reisolation frequency of the corresponding height class. Its value was calculated by re-scaling the frequency of reisolation found in the stem fragments of the class to the range $0-25$ ( $0=$ no presence; 25 , presence in all fragments of the class), so that when the fungus was reisolated in all fragments of all classes the frequency is 100\%. Letters above the columns show the results of the pairwise contrasts between the percentage of the frequencies of fungal reisolation in all stem fragments (the upper value of each column) performed within the non parametric ANOVA: values without common letters differ at the nominal $\mathrm{P}$ level $\leq 0.0018$ (actual $\mathrm{P} \leq 0.05$ level). 
compared to strains ISPaVe1018 and ISPaVe1083 (race $1,2)$ at 1 and $2 \mathrm{dpi}$. However, by $16 \mathrm{dpi}$, the highest stem level from which the fungus could be recovered was no longer dependent on the race. All strains reached a height of $75 \mathrm{~mm}$, although ISPaVe1018 did so with the greatest frequency. By 18 and $21 \mathrm{dpi}$, when symptoms of the virulent strains were obvious on all plants, both race 1,2 strains could be reisolated all along the stems, although ISPaVe1018 was faster and more continuous than ISPaVe1083. Conversely, the avirulent strain was never recovered from the highest section of the stems $(60-75 \mathrm{~mm})$ from $18 \mathrm{dpi}$ and onwards. A continuity index was established for each plant by considering the presence (1) or absence (0) of the fungus in adjacent pairs of stem sections. Generally, the distribution of fungus along the stem was discontinuous at the early time points, although more continuity was shown by race 1 (Figure 2). A peculiar pattern was shown at 8 dpi because the highest section allowing successful reisolation was lower than at 1-4 dpi (Figure 1), and the pathogen distribution was still discontinuous for all races (Figure 2). From 14 dpi onwards, colonization along the stem differed significantly between the virulent and avirulent strains, and the more extensive colonization shown by the race 1,2 strains was coupled with the appearance of obvious symptoms. In the late phase (14$21 \mathrm{dpi}$ ), continuous distribution was observed for all three strains, but for race-specific reasons (Figure 2). Both virulent strains were continuously distributed along the entire stem length, whereas the avirulent strain was continuously absent from the highest section of the stem, and continuously present in the lower

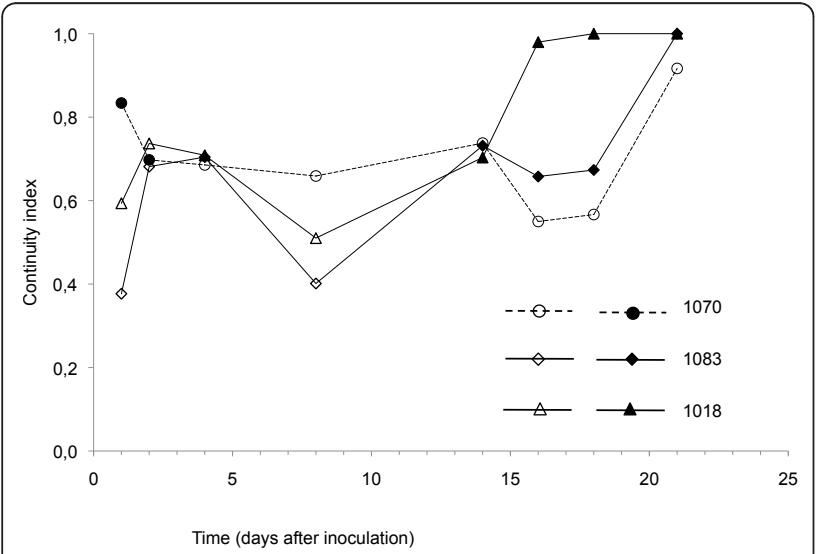

Figure 2 Distribution of the fungus along the stem expressed as continuity index, within a period of $\mathbf{2 1}$ days after inoculation. The index was determined for each plant by considering the presence (1) or absence (0) of the fungus in pairs of adjacent stem sections. The symbols are filled when the percentage of reisolation of the fungus is greater than $50 \%$, and empty when the percentage is less than $50 \%$. sections (Figure 1). Plants inoculated with race 1 remained symptomless until the end of the experiment, although the pathogen could still be reisolated. The fungus was never reisolated from uninoculated plants. All three strains could be reisolated from the stem base regardless of the time point.

\section{CDNA-AFLP analysis}

We carried out a cDNA-AFLP analysis on RNA samples from both healthy and infected melon plants to identify differentially-expressed transcripts putatively associated with the infection process and resistance response. RNA samples from the three fungal strains grown in vitro were also included in the analysis, first to help identify fungal transcripts expressed specifically in planta and second to identify fungal genes differentially expressed in vitro among the three strains. Because the fungus could be reisolated from infected stems starting from $1 \mathrm{dpi}$ in all interactions (Figure 1), samples of infected plants were collected for cDNA-AFLP analysis at 2, 4, 8 and $21 \mathrm{dpi}$. These time points were intended to take into account the early stages of infection, but also to allow the detection of pathogen transcripts when infection was well established and the mycelia produced at the late stage were abundant. RNA was also collected from uninfected plants as a control. The expression patterns of approximately 7000 transcripts were monitored with 128 different $B s t \mathrm{YI}+1 / M s e \mathrm{I}+2$ primer combinations for selective amplification. For each primer combination, 55-75 transcript derived fragments (TDFs) were visualized as bands, varying in size from 20 to $\sim 500 \mathrm{bp}$. The same average number of bands per lane was obtained from both melon and FOM colony samples (data not shown).

\section{Detection of differentially expressed transcripts in planta}

All bands were scored visually, and only those showing a 2-fold or more difference in intensity compared to uninfected controls were selected for further analysis. All bands were assigned a positive or negative score from -3 to 3 considering as zero the intensity of the corresponding band in the control lane (0: no change; $1=\sim 2$-fold change; $2=3-4$ fold change; $3=$ more than 4 -fold change). We detected a total of 1185 differentiallyexpressed TDFs.

The same scores were used to cluster profiles of differentially-expressed TDFs derived from infected melon stems. As shown in Figure 3a, FOM infection increased the abundance of a large number of mRNAs, especially in stems infected by the two race 1,2 strains at the very late phase (21 dpi), with a similar pattern of transcriptional changes. More limited changes were observed at the other time points, and also in the incompatible interaction with race 1 . 


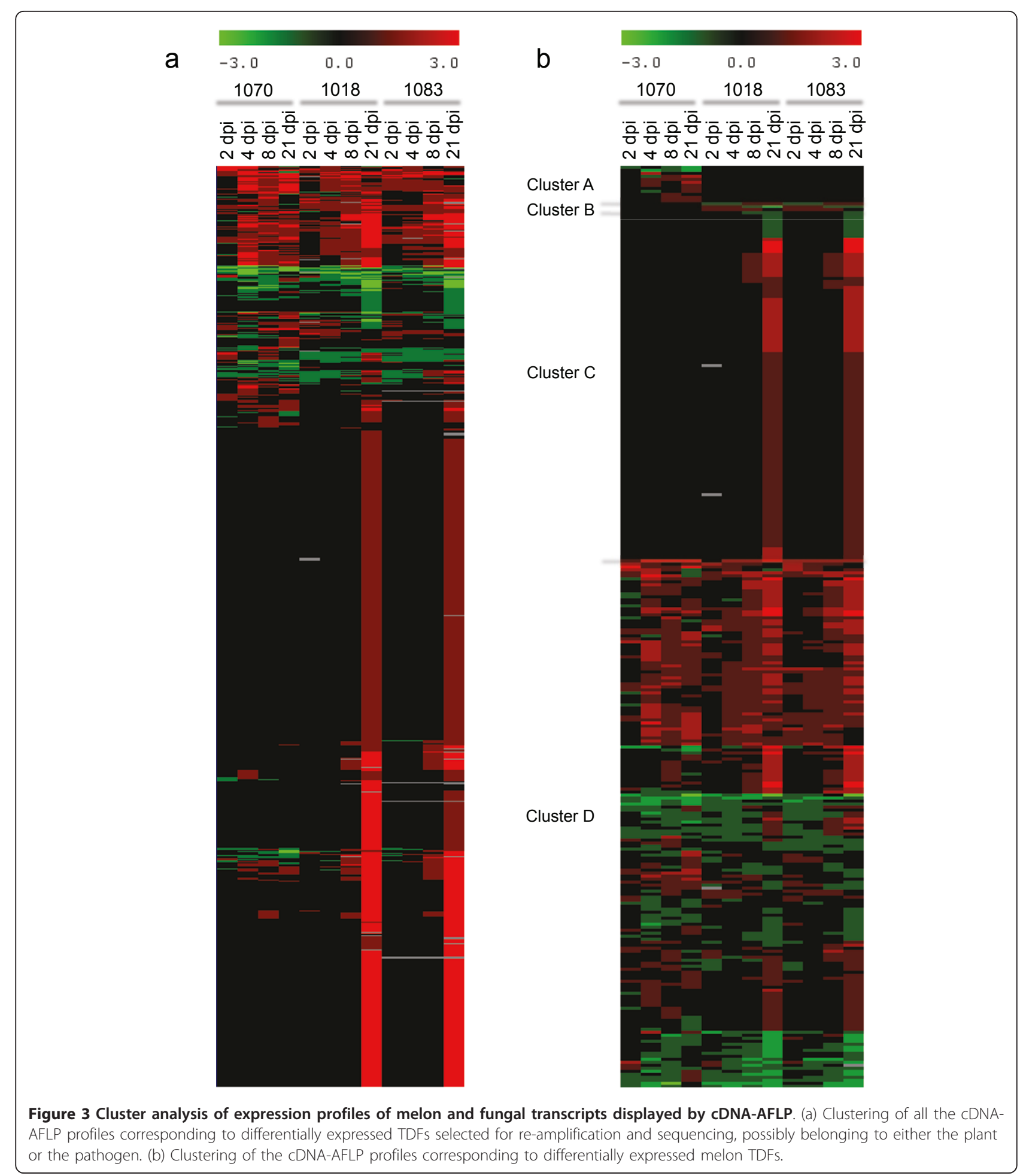

A total of 970 bands with differential expression profiles in comparison to uninfected samples in at least one interaction were excised from the gels, eluted, and reamplified with the appropriate cDNA-AFLP primers. Direct sequencing of 882 cDNA fragments yielded 627 products that could be used to screen public databases for homologous sequences, considering as significant alignments with an $\mathrm{E}<10^{-5}$.

From the BLAST analysis, 305 sequences were identified as melon transcripts (Additional File 1). Among those, 111 were found to be similar to expressed melon sequences in the vs 4.0 Melon Unigene database 
http://www.icugi.org[32] and 115 TDFs were homologous to known plant sequences in the UNIPROT [33] or NCBI [34] databases and were therefore also considered to be derived from melon. We decided another 79 sequences with no database matches were also derived from melon because a band of identical size was present in the uninfected melon sample. A total of 195 TDFs were found to be homologous to known Fusarium spp. sequences and were classed as FOM genes expressed in planta during the infection process (Additional File 2). Most of these (193) were derived from bands detected in samples infected with the virulent race 1,2 strains at $21 \mathrm{dpi}$, when the fungus had already extensively colonized the stem.

Another 127 fragments with no matches could not be assigned to either the plant or the fungus and therefore were considered as orphan TDFs. Because most of the orphan TDFs were identified at the final time point, and only in the compatible interactions, many of them are likely to represent additional fungal transcripts that currently lack functional annotations.

\section{Expression patterns and clustering of melon TDFs}

A numerical overview of the differences between incompatible and compatible interactions showing the total number of differentially-expressed melon genes at each time point and in each interaction is provided in Figure 4. At $2 \mathrm{dpi}$, it is clear that the response to both race 1 and race1,2 involves the same number of genes in all interactions, but differences emerge between the races at later time points. In the incompatible interaction, the number of modulated genes stabilizes at $\sim 125$, with a core of 54 genes that remain modulated in a coherent way (induced or repressed) until the end of the experiment (Additional File 3). Conversely, in the compatible interactions, the number of modulated genes increases similarly for both strains, with a peak of induction at $21 \mathrm{dpi}$.

To further characterize host plant responses towards the two races, we clustered the 305 modulated melon genes in four groups corresponding to: A) 11 melon sequences modulated solely during the incompatible interaction (Charentais Fom-2 inoculated with race 1); B) 3 melon sequences differentially modulated specifically in the compatible interactions at all time points in the experiment; C) 115 melon sequences modulated specifically in the compatible interactions and only at late time points (8 and $21 \mathrm{dpi}$ ); and D) 176 melon sequences repressed or induced at different stages in both the compatible and incompatible interactions (Additional File 1). These groups correspond to the clusters shown in Figure 3b. TDFs from each cluster, selected on the basis of their putative role in plantmicrobe interactions, are considered in the discussion and listed in Table 1.
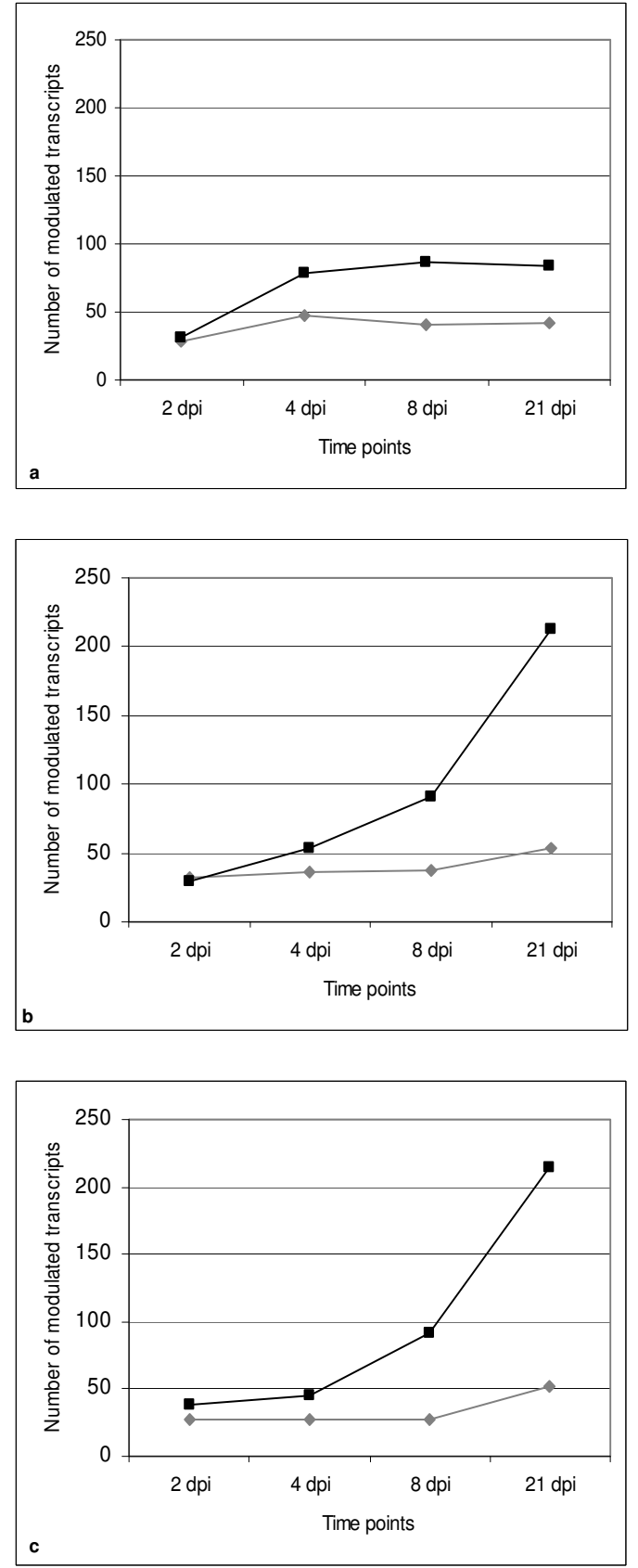

Figure 4 Total number of melon genes modulated in each interaction. Number of melon transcripts upregulated (black squares) or downregulated (gray rhombs) in the interaction with FOM race 1 strain ISPaVe1070 (a), or with FOM race 1,2 strain ISPaVe1018 (b) or strain ISPaVe1083 (c).

The number of transcripts in each cluster indicates there is little specificity in the response to compatible and incompatible interactions, since few genes are modulated solely in one type of interaction (11 are specific to the incompatible interaction, three are specific to the compatible interaction). The data also reveal a 
broad response to the virulent race 1,2 strains, specific for compatibility at late time points, and that most transcripts show variable modulation over time in either of the interactions.

The clusters also show considerable differences in the pattern of transcriptional changes. In cluster $\mathrm{C}$, almost all of the transcripts are induced. Only nine of the 115 transcripts are repressed, with perfect correspondence between the infection patterns of the race 1,2 strains. Cluster D includes variably expressed transcripts representing infections with either of the races. Most of the melon TDFs in this cluster increase or decrease coherently, in both compatible and incompatible interactions, with a small number of exceptions. Examples include an aspartokinase-homoserine dehydrogenase, a gibberellin oxidase, a protein translocase SEC61 complex gamma subunit and an AUX1-like protein, which are induced in the incompatible interaction and repressed in the compatible interactions. In contrast, a catalase isozyme 1, an ACC oxidase, a HMG-CoA reductase and a trehalase 1 show the opposite behavior.

\section{Functional categories of melon transcripts modulated by Fusarium infection}

Each transcript was functionally annotated through a careful analysis of the scientific literature and with the aid of the Gene Ontology Database [35]http://www.geneontology.org. An overview of functional categories affected by FOM infection appears more informative when each cluster is considered separately. Because Cluster A and B contain very few genes, no diagram is provided.

Cluster A contains 11 sequences, six of which have putative annotations. Among them are transcripts corresponding to a catalase involved in the response against oxidative stress and a putative calmodulin-related protein involved in signal transduction. Cluster B contains only three sequences including a transmembrane CLPTM1 family protein, which is also induced in response to bacterial infection [36] and was identified as a possible downstream target of the heat shock regulator HsfA1a [37], and a putative pyridoxal biosynthesis protein PDX1.1, which is essential for vitamin B6 biosynthesis and has been correlated to stress tolerance and photoprotection in Arabidopsis [38].

Figure 5 shows the percentages of melon genes assigned different functional categories in clusters $C$ and D. The "Metabolism" and "Unknown protein" categories are similarly represented in both clusters $(\sim 20 \%$ and $16 \%$, respectively). "Defense response" transcripts are also similarly represented with $\sim 9 \%$ and $12 \%$ in clusters $\mathrm{C}$ and D, respectively. The "Response to stimulus" and "Secondary metabolism" categories are well represented in Cluster C, each accounting for $7-8 \%$, while in cluster $\mathrm{D}$ they only represent about $2 \%$ of TDFs. The "Transport" category represents $1 \%$ of TDFs in C, but $5 \%$ in D.
Identification of $F$. oxysporum f. sp. melonis genes expressed in melon during infection

FOM genomic sequence data are scarce, so we expanded the search to include sequences from other Fusarium species or F. oxysporum formae speciales available in public databases. A total of 195 TDFs expressed in planta during the infection were identified as homologous to sequences assigned to F. oxysporum f. sp. lycopersici, F. graminearum or $F$. verticilloides (Additional File 2). Among these transcripts, 123 generated similarsized bands in the cDNA-AFLP lanes of the fungal strains grown in vitro, while the remaining 72 fragments corresponded to transcripts that were not detected in fungal colonies but only in planta during the infection and may therefore represent factors related to virulence (Figure 6a and 6b). As expected, pathogen transcripts were detected predominantly during the late infection phase and almost exclusively in the compatible interaction, probably due to the higher fungal biomass produced in host tissues. Selected FOM transcripts detected in planta are listed in Table 2.

Fungal genes expressed only in planta (Figure 6a) or in planta and in vitro (Figure 6b) were also assigned functional categories based on careful literature evaluation. This allowed us to identify some interesting differences, namely in the "Cell component" and in the "Virulence" categories, which are represented more in planta than in vitro. Other categories show similar percentages in both groups.

\section{Detection of fungal transcripts differentially expressed among strains grown in vitro}

We identified 199 bands that were differentially expressed among the three FOM strains grown in vitro, 75 of which were expressed uniquely in vitro and were selected for amplification and sequencing. For the remaining 123 TDFs, similar sized bands were also present in planta and in most cases the corresponding cDNAs had already been excised from the infected melon lanes (see above). Of the 75 TDFs expressed only in vitro, 53 were specifically expressed by strain ISPaVe1070 (race 1), and 22 (11 each) were specifically expressed by the two strains of race 1,2 (Additional File 4). Searching the Fusarium database [39] revealed sequences similar to at least one Fusarium gene for 46 fragments, 15 of which were annotated. Another 29 sequences did not match any public sequences and could represent novel $F$. oxysporum genes with a putative role in virulence.

\section{Validation of representative genes by real-time RT-PCR}

The expression profiles of seven modulated melon transcripts were analyzed by real-time RT-PCR to validate cDNA-AFLP data (Table 3). Genes were chosen among 
Table 1 Selected list of melon transcripts modulated after inoculating Fusarium oxysporum f. sp. melonis (FOM) on Charentais Fom-2 melon plants, representative of each Cluster

\begin{tabular}{|c|c|c|c|c|c|c|c|c|c|c|c|c|c|c|c|c|c|c|c|}
\hline \multirow[t]{2}{*}{ ID } & \multirow{2}{*}{$\begin{array}{l}\text { Bst- } \\
\text { Mse }\end{array}$} & \multirow{2}{*}{$\begin{array}{l}\text { Length } \\
\text { (bp) }\end{array}$} & \multirow{2}{*}{$\begin{array}{c}\text { Melon } \\
\text { Unigene } 4.0\end{array}$} & \multirow[t]{2}{*}{ Uniprot } & \multirow[t]{2}{*}{ Annotation } & \multirow{2}{*}{$\begin{array}{l}\text { Blast } \\
\text { score }\end{array}$} & \multirow[t]{2}{*}{ Category } & \multicolumn{4}{|c|}{ ISPaVe1070 } & \multicolumn{4}{|c|}{ ISPaVe1018 } & \multicolumn{4}{|c|}{ ISPaVe1083 } \\
\hline & & & & & & & & 2 & 4 & 8 & 21 & 2 & 4 & 8 & 21 & 2 & 4 & 8 & 21 \\
\hline \multicolumn{8}{|c|}{ CLUSTER A: TDFs specifically modulated in the incompatible interaction } & dpi & dpi & dpi & dpi & dpi & dpi & dpi & dpi & dpi & dpi & dpi & dpi \\
\hline P552 & CT-GT & 193 & MU45835 & P48351 & Catalase isozyme 3 & $2 \mathrm{E}-80$ & $\begin{array}{l}\text { Defence } \\
\text { response }\end{array}$ & 0 & 0 & 1 & 0 & 0 & 0 & 0 & 0 & 0 & 0 & 0 & 0 \\
\hline P355 & CG-CC & 153 & MU54667 & - & putative calmodulin-related protein & $3 E-41$ & $\begin{array}{l}\text { Signal } \\
\text { transduction }\end{array}$ & 0 & 1 & 1 & 1 & 0 & 0 & 0 & 0 & 0 & 0 & 0 & 0 \\
\hline P1257 & TG-AA & 119 & MU54228 & - & $\begin{array}{l}\text { Protein translocase SEC61 complex gamma } \\
\text { subunit }\end{array}$ & $4 \mathrm{E}-40$ & Transport & 0 & -1 & 0 & 1 & 0 & 0 & 0 & 0 & 0 & 0 & 0 & 0 \\
\hline \multicolumn{20}{|c|}{ CLUSTER B: TDFs specifically modulated in the compatible interaction at all time points } \\
\hline P431 & CC-TC & 254 & - & Q69LA6 & Probable pyridoxal biosynthesis protein PDX1.1. & $2 \mathrm{E}-28$ & Metabolism & 0 & 0 & 0 & 0 & 1 & 1 & -1 & -2 & 1 & 1 & -1 & -1 \\
\hline P75 & ТT-TA & 248 & MU54623 & - & Transmembrane CLPTM1 family protein & 7E-56 & $\begin{array}{l}\text { Signal } \\
\text { transduction }\end{array}$ & 0 & 0 & 0 & 0 & -1 & -1 & 1 & 1 & -1 & -1 & 1 & 1 \\
\hline \multicolumn{20}{|c|}{ CLUSTER C: TDFs specifically modulated in the compatible interaction, only at late time points ( 8 and $21 \mathrm{dpi})$} \\
\hline P554 & CT-GT & 210 & MU45886 & P09918 & 13S-lipoxygenase & $2 \mathrm{E}-27$ & $\begin{array}{l}\text { Defence } \\
\text { response }\end{array}$ & 0 & 0 & 0 & 0 & 0 & 0 & 0 & 2 & 0 & 0 & 0 & 3 \\
\hline P767 & $\begin{array}{l}\text { CA- } \\
\text { CG }\end{array}$ & 115 & MU47701 & Q39799 & Endochitinase 1 precursor & $2 \mathrm{E}-11$ & $\begin{array}{l}\text { Defence } \\
\text { response }\end{array}$ & 0 & 0 & 0 & 0 & 0 & 0 & 1 & 2 & 0 & 0 & 1 & 2 \\
\hline P1190 & CA-GT & 402 & MU45840 & - & type I proteinase inhibitor-like protein & $1 \mathrm{E}-123$ & $\begin{array}{l}\text { Defence } \\
\text { response }\end{array}$ & 0 & 0 & 0 & 0 & 0 & 0 & 0 & 1 & 0 & 0 & 0 & 1 \\
\hline P763 & CA-CC & 167 & - & Q8S0S6 & Gibberellin 2-oxidase & $3 \mathrm{E}-12$ & Metabolism & 0 & 0 & 0 & 0 & 0 & 0 & 0 & 1 & 0 & 0 & 0 & 1 \\
\hline P962 & CT-TT & 175 & MU48285 & - & 12-oxophytodienoate reductase & $3 \mathrm{E}-27$ & Metabolism & 0 & 0 & 0 & 0 & 0 & 0 & 1 & 2 & 0 & 0 & 1 & 2 \\
\hline P257 & TT-CA & 354 & MU52195 & Q75GKO & IAA type protein & $E+00$ & $\begin{array}{l}\text { Response to } \\
\text { stimulus }\end{array}$ & 0 & 0 & 0 & 0 & 0 & 0 & 1 & 2 & 0 & 0 & 1 & 2 \\
\hline P1011 & $\Pi \pi-G A$ & 118 & - & Q9LSQ4 & Indole-3-acetic acid-amido synthetase GH3.6 & $2 \mathrm{E}-16$ & $\begin{array}{l}\text { Response to } \\
\text { stimulus }\end{array}$ & 0 & 0 & 0 & 0 & 0 & 0 & 0 & 1 & 0 & 0 & 0 & 1 \\
\hline P1236 & CA-AT & 53 & MU51174 & - & xanthine dehydrogenase-like protein & $5 \mathrm{E}-19$ & $\begin{array}{l}\text { Response to } \\
\text { stimulus }\end{array}$ & 0 & 0 & 0 & 0 & 0 & 0 & 0 & 2 & 0 & 0 & 0 & 2 \\
\hline P1409 & TG-CA & 305 & - & O24174 & Betaine aldehyde dehydrogenase (BADH). & $2 \mathrm{E}-18$ & $\begin{array}{l}\text { Response to } \\
\text { stimulus }\end{array}$ & 0 & 0 & 0 & 0 & 0 & 0 & 0 & 1 & 0 & 0 & 0 & 1 \\
\hline P1430 & TA-CC & 422 & - & Q9S795 & $\begin{array}{l}\text { Betaine aldehyde dehydrogenase 1, chloroplast } \\
\text { precursor (BADH) }\end{array}$ & $4 \mathrm{E}-23$ & $\begin{array}{l}\text { Response to } \\
\text { stimulus }\end{array}$ & 0 & 0 & 0 & 0 & 0 & 0 & 0 & 1 & 0 & 0 & 0 & 1 \\
\hline P1418 & TG-CC & 378 & MU65227 & B9RFG8 & calmodulin-binding protein, putative, expressed & $5 E-113$ & $\begin{array}{l}\text { Signal } \\
\text { transduction }\end{array}$ & 0 & 0 & 0 & 0 & 0 & 0 & 0 & 1 & 0 & 0 & 0 & 1 \\
\hline P1479 & TC-TC & 278 & MU45785 & P93087 & Calmodulin (CaM) & $3 E-129$ & $\begin{array}{l}\text { Signal } \\
\text { transduction }\end{array}$ & 0 & 0 & 0 & 0 & 0 & 0 & 0 & 1 & 0 & 0 & 0 & 1 \\
\hline \multicolumn{20}{|c|}{ CLUSTER D: TDFs repressed or induced at different stages in both the compatible and incompatible interactions } \\
\hline P200 & TA-TA & 445 & MU48195 & Q08506 & ACC oxidase & $E+00$ & $\begin{array}{l}\text { Defence } \\
\text { response }\end{array}$ & 1 & 2 & 1 & 0 & 1 & 1 & 1 & 1 & 1 & 1 & 1 & 1 \\
\hline P413 & CC-TT & 214 & - & B9R992 & ACC oxidase 1 & $2 \mathrm{E}-09$ & $\begin{array}{l}\text { Defence } \\
\text { response }\end{array}$ & 2 & -1 & -1 & 0 & 1 & -1 & -1 & -2 & 1 & -1 & -1 & -2 \\
\hline P449 & CC-TG & 155 & - & Q43419 & ACC oxidase. & $7 \mathrm{E}-17$ & $\begin{array}{l}\text { Defence } \\
\text { response }\end{array}$ & 1 & -1 & 0 & -1 & 0 & -1 & 0 & 0 & 1 & 0 & 0 & 0 \\
\hline
\end{tabular}


Table 1 Selected list of melon transcripts modulated after inoculating Fusarium oxysporum f. sp. melonis (FOM) on Charentais Fom-2 melon plants, representative of each Cluster (Continued)

\begin{tabular}{|c|c|c|c|c|c|c|c|c|c|c|c|c|c|c|c|c|c|c|c|}
\hline P640 & $\begin{array}{l}C G- \\
A C\end{array}$ & 325 & - & Q40778 & Allene oxide synthase & $3 \mathrm{E}-24$ & $\begin{array}{l}\text { Defence } \\
\text { response }\end{array}$ & 0 & -1 & 1 & 2 & -1 & 0 & 1 & 2 & 0 & 0 & 1 & 1 \\
\hline P870 & $\mathrm{CT}-\mathrm{AG}$ & 407 & - & Q8H1S4 & ACC oxidase homolog 4 & $3 \mathrm{E}-26$ & $\begin{array}{l}\text { Defence } \\
\text { response }\end{array}$ & 0 & 0 & 0 & -1 & 0 & 0 & 0 & 1 & 0 & 0 & 0 & 1 \\
\hline P1109 & CG-GT & 206 & MU47271 & - & Catalase isozyme 1 & $2 \mathrm{E}-92$ & $\begin{array}{l}\text { Defence } \\
\text { response }\end{array}$ & 0 & 0 & 0 & -1 & 0 & 0 & 0 & 1 & 0 & 0 & 0 & 1 \\
\hline P285 & $\Pi \mathrm{T}-\mathrm{CC}$ & 144 & MU45886 & B7FDE5 & 135-lipoxygenase & $3 E-29$ & $\begin{array}{l}\text { Defence } \\
\text { response }\end{array}$ & -1 & -1 & 0 & 1 & -1 & -1 & 1 & 0 & -1 & -1 & 1 & 1 \\
\hline P871 & CT-AG & 390 & - & Q9SFB5 & Serine carboxypeptidase-like 27 precursor & $7 \mathrm{E}-56$ & $\begin{array}{l}\text { Defence } \\
\text { response }\end{array}$ & -1 & -1 & -1 & 1 & -1 & -1 & -1 & -1 & 0 & -1 & -1 & -1 \\
\hline P1327 & $\mathrm{TC}-\mathrm{CG}$ & 214 & MU47122 & Q9SQX6 & Peptidase S10, serine carboxypeptidase & $5 \mathrm{E}-81$ & $\begin{array}{l}\text { Defence } \\
\text { response }\end{array}$ & 0 & 1 & 0 & 0 & 0 & 0 & 0 & 1 & 0 & 0 & 0 & 1 \\
\hline P534 & CT-GA & 147 & - & A7NSX3 & Endo 1,4 beta glucanase & $1 \mathrm{E}-09$ & $\begin{array}{l}\text { Defence } \\
\text { response }\end{array}$ & 0 & -1 & -1 & 0 & -1 & -1 & -1 & -2 & 0 & 0 & 0 & -1 \\
\hline P155 & TG-GG & 172 & MU55410 & - & aspartokinase-homoserine dehydrogenase & $2 \mathrm{E}-70$ & Metabolism & 0 & 1 & 0 & 1 & 0 & 0 & -1 & 0 & 0 & 0 & -1 & 0 \\
\hline P273 & $\mathrm{TT}-\mathrm{CT}$ & 83 & MU45201 & - & HMG-CoA reductase & $1 \mathrm{E}-05$ & Metabolism & 0 & 0 & 0 & -1 & 0 & 1 & 1 & 3 & 0 & 1 & 1 & 3 \\
\hline P517 & $\mathrm{CT}-\mathrm{CG}$ & 134 & - & P68173 & $\begin{array}{l}\text { Adenosylhomocysteinase (S-adenosyl-L- } \\
\text { homocysteine hydrolase) }\end{array}$ & $2 \mathrm{E}-13$ & Metabolism & 0 & -1 & -1 & -1 & 0 & 0 & 0 & 0 & 0 & 0 & 0 & -1 \\
\hline P996b & CT-TG & 151 & - & Q9SP37 & Adenosylhomocysteise & $8 \mathrm{E}-15$ & Metabolism & 0 & -1 & -1 & -1 & 0 & 0 & 0 & -1 & 0 & 0 & 0 & -1 \\
\hline P1336 & $T C-G A$ & 332 & - & Q39110 & Gibberellin 20 oxidase 1 & $2 \mathrm{E}-45$ & Metabolism & 1 & 0 & 0 & 1 & 0 & 0 & 0 & -1 & 0 & 0 & 0 & -1 \\
\hline P633 & $\begin{array}{l}\text { CG- } \\
\text { AA }\end{array}$ & 190 & - & B9GL14 & Trehalase 1 GMTRE1 & $3 \mathrm{E}-19$ & $\begin{array}{l}\text { Response to } \\
\text { stimulus }\end{array}$ & -1 & 0 & 0 & 0 & 0 & 0 & 1 & 2 & 0 & 0 & 1 & 2 \\
\hline P86 & $\Pi T-\Pi$ & 167 & MU58530 & - & NDR1-like protein & $2 \mathrm{E}-51$ & $\begin{array}{l}\text { Signal } \\
\text { transduction }\end{array}$ & 1 & 1 & 0 & 0 & 0 & 0 & 1 & 0 & 0 & 0 & 1 & 0 \\
\hline P204 & TA-TA & 384 & MU46993 & P43187 & Ca2+-binding protein & $0 \mathrm{E}+00$ & $\begin{array}{l}\text { Signal } \\
\text { transduction }\end{array}$ & 0 & 2 & 1 & 2 & 0 & 0 & 1 & 2 & 0 & 0 & 1 & 2 \\
\hline P251 & TA-CA & 64 & MU52101 & - & protein kinase family protein & $2 \mathrm{E}-24$ & $\begin{array}{l}\text { Signal } \\
\text { transduction }\end{array}$ & 0 & 1 & 1 & 1 & -1 & 0 & 0 & -1 & 1 & 1 & -1 & -1 \\
\hline P873 & $\mathrm{CT}-\mathrm{AG}$ & 360 & - & Q9ZTX8 & Auxin response factor 6 & $2 \mathrm{E}-19$ & $\begin{array}{l}\text { Signal } \\
\text { transduction }\end{array}$ & 0 & -1 & 0 & 1 & 0 & 0 & -1 & -1 & 0 & 0 & -1 & -1 \\
\hline P1370 & TC-GC & 222 & MU50486 & Q9FKW4 & calcium-dependent protein kinase CDPK1444 & $1 \mathrm{E}-112$ & $\begin{array}{l}\text { Signal } \\
\text { transduction }\end{array}$ & 0 & 2 & 1 & 1 & 0 & 1 & 1 & 1 & 1 & 1 & 1 & 0 \\
\hline P1393 & TA-GC & 247 & MU51221 & - & $\begin{array}{l}\text { calcium-dependent calmodulin-independent } \\
\text { protein kinase CDPK }\end{array}$ & $3 \mathrm{E}-18$ & $\begin{array}{l}\text { Signal } \\
\text { transduction }\end{array}$ & 0 & 1 & 0 & 0 & 0 & 1 & 1 & 1 & 0 & 1 & 1 & 1 \\
\hline P1478 & $\mathrm{TC}-\mathrm{TC}$ & 269 & - & Q9LFL1 & Receptor protein kinase-like protein & $2 \mathrm{E}-20$ & $\begin{array}{l}\text { Signal } \\
\text { transduction }\end{array}$ & 0 & 2 & 1 & 1 & 0 & 0 & 1 & 2 & 0 & 0 & 1 & 2 \\
\hline P1155 & CC-AA & 108 & - & Q37145 & $\begin{array}{l}\text { Calcium-transporting ATPase 1, chloroplast } \\
\text { precursor }\end{array}$ & $7 \mathrm{E}-10$ & $\begin{array}{l}\text { Signal } \\
\text { transduction }\end{array}$ & 0 & 1 & 1 & 2 & 0 & 0 & 1 & 0 & 0 & 0 & 0 & 0 \\
\hline P57 & TT-AC & 237 & - & Q8H0E1 & AUX1-like auxin transport protein & $4 \mathrm{E}-11$ & Transport & 1 & 1 & 2 & 2 & 0 & 0 & -1 & -1 & 0 & 0 & 0 & -1 \\
\hline P1100 & $\begin{array}{l}\text { CG- } \\
\text { GA }\end{array}$ & 105 & MU54228 & Q9SW34 & $\begin{array}{l}\text { Protein translocase SEC61 complex gamma } \\
\text { subunit }\end{array}$ & $2 E-44$ & Transport & 0 & 2 & 0 & 1 & -2 & -1 & -1 & -2 & -1 & -1 & 0 & -1 \\
\hline
\end{tabular}

This selection of representative genes modulated by infection with avirulent (ISPaVe1070 race1) or virulent (ISPaVe1018 and ISPaVe1083 race 1,2) strains of FOM is divided into clusters as defined in the results section. Identification numbers (ID) correspond to progressive numbering of bands in the gels. The primer combination used to visualize each band (Bst-Mse column) and the corresponding length of the fragment are reported, along with the accession number in the database from which the sequence was retrieved, its annotation, the corresponding BLAST score and the functional category to which each transcript was assigned. In addition, the table shows the expression profile of each transcript, in each interaction and at each time point, estimated on the basis of the band intensity with a score from -3 to 3 , in comparison to the corresponding band in the uninfected controls. 


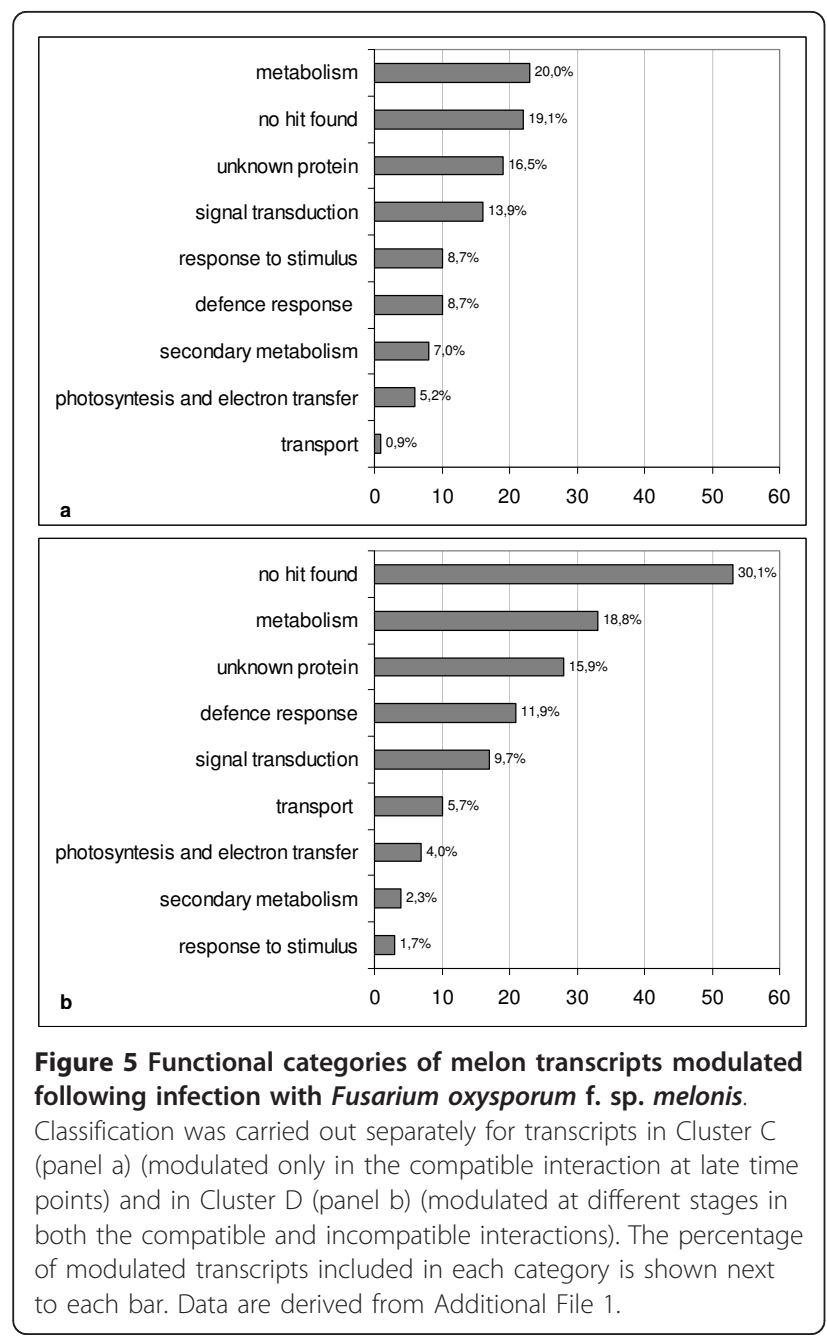

those expressed in planta as representative of the different clusters, with a preference for defense-related genes. The RT-PCR expression profiles of six genes agreed strongly with the cDNA-AFLP data, whereas the protein translocase SEC61 complex gamma subunit appeared to be induced to a significantly greater extent according to the RT-PCR results. The identity and expression of five fungal TDFs expressed in planta was confirmed using the same technique (Table 4). Minor discrepancies are observed when expression levels are measured using different techniques $[40,41]$ but the RT-PCR and cDNAAFLP data are largely concordant, confirming the reliability of the results.

\section{Discussion}

Melon is an important horticultural crop and is rapidly becoming a popular research model for cucurbits, thanks to the increasing availability of genomic resources. Several diseases affect melon production, but

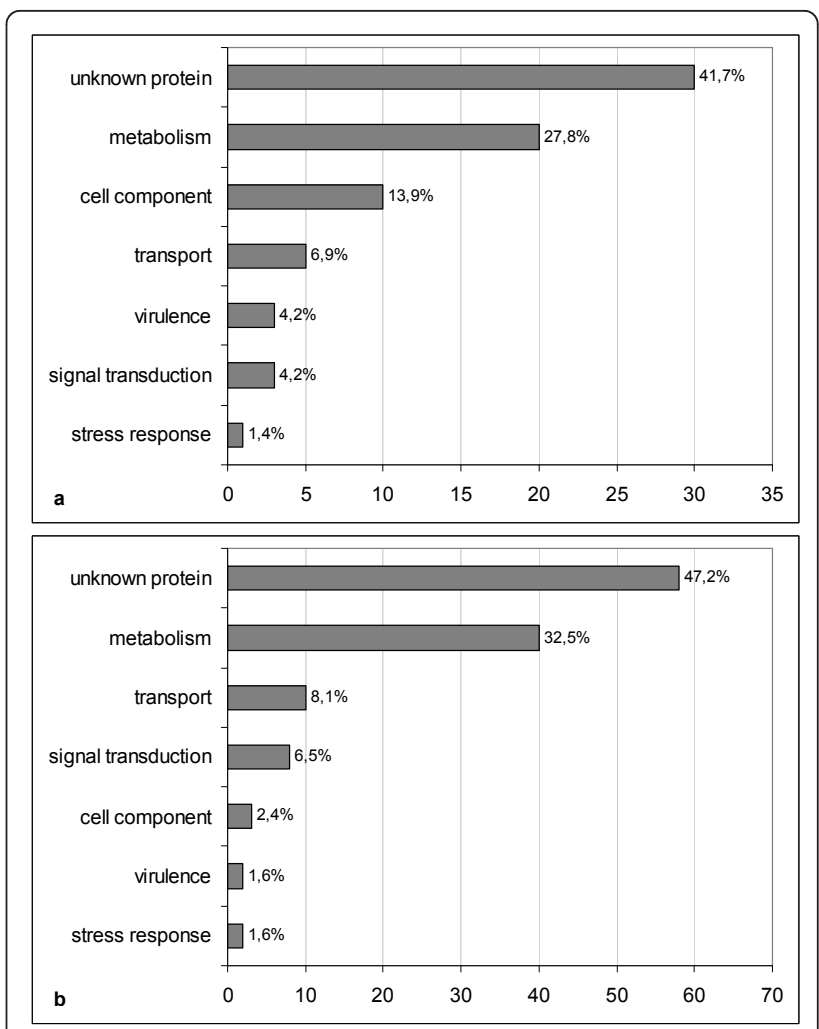

Figure 6 Functional categories of fungal transcripts expressed in infected melon plants. Classification of Fusarium oxysporum $\mathrm{f}$. sp. melonis transcripts has been performed separately for those expressed only in planta (panel a) or both in planta and in vitro (panel b). The percentage of modulated transcripts included in each category is shown next to each bar. Data are derived from Additional File 2.

molecular investigations of plant-pathogen interactions in melon are still rare [42]. Recently, a melon array resource has been developed, allowing the transcriptomic analysis of several physiological and pathological conditions [43]. However, the melon genome has not been fully sequenced, so alternative transcriptomic approaches allowing novel gene discovery are still important. The cDNA-AFLP method is particularly appropriate for gene expression studies in non-model species and it also allows identification of both plant and pathogen genes expressed during infection, an important pre-requisite for the identification of pathogenicity and virulence factors and thus for the identification of targeted control strategies [44-48,20]. Our study provides the first large-scale investigation of gene expression changes that occur when melon is infected with FOM, the most important melon pathogen, and is the first to compare compatible and incompatible interactions in the same genetic background (Charentais Fom-2). 
Table 2 Selected list of Fusarium oxysporum f. sp. melonis (FOM) transcripts expressed in melon plants during infection

\begin{tabular}{|c|c|c|c|c|c|c|c|c|c|c|c|c|c|c|c|c|c|c|}
\hline \multirow[t]{2}{*}{ ID } & \multirow{2}{*}{$\begin{array}{l}\text { Bst- } \\
\text { Mse }\end{array}$} & \multirow{2}{*}{$\begin{array}{l}\text { Length } \\
\text { (bp) }\end{array}$} & \multirow[t]{2}{*}{ Accessions } & \multirow[t]{2}{*}{ Annotation } & \multirow{2}{*}{$\begin{array}{l}\text { Blast } \\
\text { score }\end{array}$} & \multirow[t]{2}{*}{ Category } & \multicolumn{4}{|c|}{ ISPaVe1070 } & \multicolumn{4}{|c|}{ ISPaVe1018 } & \multicolumn{4}{|c|}{ ISPaVe1083 } \\
\hline & & & & & & & $\begin{array}{c}2 \\
\mathrm{dpi}\end{array}$ & $\begin{array}{c}4 \\
\mathrm{dpi}\end{array}$ & $\begin{array}{c}8 \\
\mathrm{dpi}\end{array}$ & $\begin{array}{l}21 \\
\text { dpi }\end{array}$ & $\begin{array}{c}2 \\
\mathrm{dpi}\end{array}$ & $\begin{array}{c}4 \\
\mathrm{dpi}\end{array}$ & $\begin{array}{c}8 \\
\mathrm{dpi}\end{array}$ & $\begin{array}{l}21 \\
\text { dpi }\end{array}$ & $\begin{array}{c}2 \\
\mathrm{dpi}\end{array}$ & $\begin{array}{c}4 \\
\mathrm{dpi}\end{array}$ & $\begin{array}{c}8 \\
\text { dpi }\end{array}$ & $\begin{array}{l}21 \\
\mathrm{dpi}\end{array}$ \\
\hline \multicolumn{19}{|c|}{ TDFs expressed only in planta } \\
\hline P1425 & TG-CG & 277 & FOXT_01463 & $\begin{array}{l}\text { hypothetical protein } \\
\text { similar to actin } \\
\text { binding protein }\end{array}$ & $3 E-76$ & $\begin{array}{l}\text { cell } \\
\text { component }\end{array}$ & 0 & 0 & 0 & 0 & 0 & 0 & 0 & 2 & 0 & 0 & 0 & 2 \\
\hline P1443 & $\mathrm{TA}-\mathrm{CG}$ & 190 & FOXG_00066 & dynamin-2 & $6 \mathrm{E}-76$ & $\begin{array}{l}\text { cell } \\
\text { component }\end{array}$ & 0 & 0 & 0 & 0 & 0 & 0 & 0 & 1 & 0 & 0 & 0 & 1 \\
\hline P406 & CC-TT & 479 & FOXT_05654 & $\begin{array}{l}\text { hypothetical protein } \\
\text { similar to } 1,4 \text {-beta- } \\
\text { glucanase }\end{array}$ & $7 \mathrm{E}-124$ & cell wall & 0 & 0 & 0 & 0 & 0 & 0 & 0 & 2 & 0 & 0 & 0 & 1 \\
\hline P998 & CT-TG & 174 & FOXT_00113 & chitin synthase 4 & $3 \mathrm{E}-52$ & cell wall & 0 & 0 & 0 & 0 & 0 & 0 & 0 & 1 & 0 & 0 & 0 & 1 \\
\hline P1261 & TG-AT & 209 & AF080485 & $\begin{array}{l}\text { Fusarium oxysporum } \\
\text { f. sp. lycopersici } \\
\text { pectate lyase }\end{array}$ & $2 \mathrm{E}-87$ & $\begin{array}{l}\text { cell wall- } \\
\text { degrading }\end{array}$ & 0 & 0 & 0 & 0 & 0 & 0 & 0 & 1 & 0 & 0 & 0 & 2 \\
\hline P553 & $\mathrm{CT}-\mathrm{GT}$ & 235 & FOXG_09638 & $\begin{array}{l}\text { endo-1,4-beta- } \\
\text { xylanase } 2 \text { precursor }\end{array}$ & 7E-101 & $\begin{array}{l}\text { cell wall- } \\
\text { degrading }\end{array}$ & 0 & 0 & 0 & 1 & 0 & 0 & 0 & 0 & 0 & 0 & 0 & 0 \\
\hline P1362 & TC-GC & 377 & FOXT_13415 & $\begin{array}{l}\text { endo-1,4-beta- } \\
\text { xylanase }\end{array}$ & $9 \mathrm{E}-145$ & $\begin{array}{l}\text { cell wall- } \\
\text { degrading }\end{array}$ & 0 & 0 & 0 & 0 & 0 & 0 & 0 & 2 & 0 & 0 & 0 & 1 \\
\hline P32 & TT-AA & 148 & FOXG_06061 & squalene synthetase & $1 \mathrm{E}-42$ & metabolism & 0 & 0 & 0 & 0 & 0 & 0 & 0 & 1 & 0 & 0 & 0 & 1 \\
\hline P675 & CG-TT & 259 & FOXT_08412 & $\begin{array}{l}\text { protein similar to } \\
\text { phosphoserine } \\
\text { phosphatase }\end{array}$ & $1 \mathrm{E}-114$ & $\begin{array}{l}\text { signal } \\
\text { transduction }\end{array}$ & 0 & 0 & 0 & 0 & 0 & 0 & 0 & 1 & 0 & 0 & 0 & 1 \\
\hline P1473 & TG-AG & 224 & FOXT_00565 & $\begin{array}{l}\text { hypothetical protein } \\
\text { similar to calnexin }\end{array}$ & $4 \mathrm{E}-102$ & $\begin{array}{l}\text { signal } \\
\text { transduction }\end{array}$ & 0 & 0 & 0 & 0 & 0 & 0 & 0 & 1 & 0 & 0 & 0 & 1 \\
\hline P842 & CT-AA & 382 & FOXG_03102 & $\begin{array}{l}\text { hypothetical protein } \\
\text { similar to MADS box } \\
\text { protein }\end{array}$ & $4 \mathrm{E}-131$ & $\begin{array}{l}\text { signal } \\
\text { transduction }\end{array}$ & 0 & 0 & 1 & 1 & 0 & 1 & 1 & 2 & 0 & 1 & 1 & 2 \\
\hline P1331 & TC-CG & 165 & FVET_00351 & $\begin{array}{l}\text { hypothetical protein } \\
\text { similar to vps } 28 \\
\text { protein }\end{array}$ & $3 E-24$ & transport & 0 & 1 & 0 & 0 & 0 & 0 & 0 & 1 & 0 & 0 & 0 & 1 \\
\hline P888 & TC-TA & 449 & FOXT_11289 & $\begin{array}{l}\text { plasma membrane } \\
\text { ATPase }\end{array}$ & $1 \mathrm{E}-178$ & transport & 0 & 0 & 0 & 0 & 0 & 0 & 0 & 1 & 0 & 0 & 0 & 1 \\
\hline P1014 & $\Pi$ T-GA & 140 & FOXT_12267 & $\begin{array}{l}\text { hypothetical protein } \\
\text { similar to hexose } \\
\text { transporter }\end{array}$ & $8 \mathrm{E}-25$ & transport & 0 & 0 & 0 & 0 & 0 & 0 & 0 & 2 & 0 & 0 & 0 & 1 \\
\hline P1066 & CC-GC & 380 & FOXT_11113 & $\begin{array}{l}\text { mitochondrial } \\
\text { phosphate carrier } \\
\text { protein }\end{array}$ & $1 \mathrm{E}-177$ & transport & 0 & 0 & 0 & 0 & 0 & 0 & 0 & 1 & 0 & 0 & 0 & 1 \\
\hline P1232 & CA-AT & 341 & FOXT_10521 & $\begin{array}{l}\text { mitochondrial import } \\
\text { inner membrane } \\
\text { translocase }\end{array}$ & $1 \mathrm{E}-63$ & transport & 0 & 0 & 0 & 0 & 0 & 0 & 0 & 1 & 0 & 0 & 0 & 1 \\
\hline P819 & TC-AC & 359 & FOXG_09125 & $\begin{array}{l}\text { hypothetical protein } \\
\text { similar to } \\
\text { avenacinase }\end{array}$ & $6 \mathrm{E}-117$ & virulence & 0 & 0 & 0 & 0 & 0 & 0 & 0 & 3 & 0 & 0 & 0 & 1 \\
\hline P1258 & TG-AT & 468 & FOXG_09782 & $\begin{array}{l}\text { siderophore iron } \\
\text { transporter mirB }\end{array}$ & 0 & virulence & 0 & 0 & 0 & 0 & 0 & 0 & 0 & 1 & 0 & 0 & 0 & 1 \\
\hline P1482 & TC-TC & 187 & FOXT_03354 & $\begin{array}{l}\text { hypothetical protein } \\
\text { similar to Fum16p }\end{array}$ & $3 \mathrm{E}-81$ & virulence & 0 & 0 & 0 & 0 & 0 & 0 & 0 & 2 & 0 & 0 & 0 & 2 \\
\hline
\end{tabular}

TDFs expressed both in planta and in vitro

\begin{tabular}{|c|c|c|c|c|c|c|c|c|c|c|c|c|c|c|c|c|c|c|}
\hline P417 & CC-TT & 168 & FOXT_11103 & $\begin{array}{l}\text { hypothetical protein } \\
\text { similar to sterol } \\
\text { esterase precursor }\end{array}$ & $6 \mathrm{E}-57$ & $\begin{array}{l}\text { lipid } \\
\text { metabolism }\end{array}$ & 0 & 0 & 0 & 0 & 0 & 0 & 0 & 2 & 0 & 0 & 0 & 1 \\
\hline P292 & TT-CG & 180 & FOXT_00536 & $\begin{array}{l}\text { UTP-glucose-1- } \\
\text { phosphate } \\
\text { uridylyltransferase }\end{array}$ & $1 \mathrm{E}-77$ & metabolism & 0 & 0 & 0 & 0 & 0 & 0 & 0 & 2 & 0 & 0 & 2 & \\
\hline P305 & $\begin{array}{l}\text { CG- } \\
\text { CA }\end{array}$ & 199 & FOXG_09447 & $\begin{array}{l}\text { pyruvate } \\
\text { dehydrogenase E1 } \\
\text { component subunit } \\
\text { alpha }\end{array}$ & $2 \mathrm{E}-88$ & metabolism & 0 & 0 & 0 & 0 & 0 & 0 & 0 & 2 & 0 & 0 & 0 & 1 \\
\hline
\end{tabular}


Table 2 Selected list of Fusarium oxysporum f. sp. melonis (FOM) transcripts expressed in melon plants during infection (Continued)

\begin{tabular}{|c|c|c|c|c|c|c|c|c|c|c|c|c|c|c|c|c|c|c|}
\hline P344 & CG-CC & 363 & FOXG_11520 & $\begin{array}{l}\text { NAD-specific } \\
\text { glutamate } \\
\text { dehydrogenase }\end{array}$ & $1 \mathrm{E}-103$ & metabolism & 0 & 0 & 0 & 0 & 0 & 0 & 0 & 3 & 0 & 0 & 0 & 2 \\
\hline P1211 & $\begin{array}{l}\text { CA- } \\
\text { GC }\end{array}$ & 159 & FOXG_10419 & $\begin{array}{l}\text { malate } \\
\text { dehydrogenase, } \\
\text { mitochondrial } \\
\text { precursor }\end{array}$ & $\begin{array}{c}1,00 \mathrm{E}- \\
67\end{array}$ & metabolism & 0 & 0 & 0 & 0 & 0 & 0 & 0 & 2 & 0 & 0 & 0 & 2 \\
\hline P1333 & TC-CG & 140 & FOXT_11398 & $\begin{array}{l}\text { UDP-glucose 6- } \\
\text { dehydrogenase }\end{array}$ & $\begin{array}{c}4,00 \mathrm{E}- \\
58\end{array}$ & metabolism & 0 & 0 & 0 & 0 & 0 & 0 & 0 & 3 & 0 & 0 & 0 & 3 \\
\hline P1486 & CC-CA & 226 & FOXT_07937 & $\begin{array}{l}\text { glutathione } \\
\text { reductase }\end{array}$ & $1 \mathrm{E}-89$ & metabolism & 0 & 0 & 0 & 0 & 0 & 0 & 0 & 1 & 0 & 0 & 0 & 1 \\
\hline P1280 & TG-TC & 358 & FOXT_00266 & $\begin{array}{l}\text { hypothetical protein } \\
\text { similar to } \\
\text { multiubiquitin }\end{array}$ & $1 \mathrm{E}-60$ & $\begin{array}{l}\text { protein } \\
\text { metabolism }\end{array}$ & 0 & 0 & 0 & 0 & 0 & 0 & 0 & 1 & 0 & 0 & 0 & 1 \\
\hline P201 & TA-TA & 443 & FOXG_03710 & $\begin{array}{l}\text { hypothetical protein } \\
\text { similar to } \\
\text { proteasome } \\
\text { regulatory }\end{array}$ & 0 & $\begin{array}{l}\text { protein } \\
\text { metabolism }\end{array}$ & 0 & 0 & 0 & 0 & 0 & 0 & 0 & 1 & 0 & 0 & 0 & 1 \\
\hline P1398 & TA-GG & 286 & FOXT_00231 & $\begin{array}{l}\text { proteasome } \\
\text { component C5 }\end{array}$ & $2 \mathrm{E}-139$ & $\begin{array}{l}\text { protein } \\
\text { metabolism }\end{array}$ & 0 & 0 & 0 & 0 & 0 & 0 & 0 & 1 & 0 & 0 & 0 & 1 \\
\hline P304 & $\begin{array}{l}\text { CG- } \\
\text { CA }\end{array}$ & 215 & FOXT_10713 & $\begin{array}{l}\text { hypothetical protein } \\
\text { similar to Ca2+ } \\
\text { ATPase }\end{array}$ & $4 \mathrm{E}-68$ & $\begin{array}{l}\text { signal } \\
\text { transduction }\end{array}$ & 0 & 0 & 0 & 0 & 0 & 0 & 0 & 1 & 0 & 0 & 0 & 1 \\
\hline P960 & CT-Tा & 187 & FOXG_05517 & $\begin{array}{l}\text { CAMP-dependent } \\
\text { protein kinase } \\
\text { regulatory subunit }\end{array}$ & $6 \mathrm{E}-79$ & $\begin{array}{l}\text { signal } \\
\text { transduction }\end{array}$ & 0 & 0 & 0 & 0 & 0 & 0 & 0 & 1 & 0 & 0 & 0 & 1 \\
\hline P469 & $\mathrm{CT}-\mathrm{CA}$ & 294 & FOXT_12260 & peroxidase/catalase 2 & $7 \mathrm{E}-148$ & $\begin{array}{l}\text { stress } \\
\text { response }\end{array}$ & 0 & 0 & 0 & 0 & 0 & 0 & 0 & 2 & 0 & 0 & 0 & 2 \\
\hline P1134 & $\begin{array}{l}\text { CG- } \\
\text { GG }\end{array}$ & 352 & FOXT_01866 & sorting nexin-41 & 0 & transport & 0 & 0 & 0 & 0 & 0 & 0 & 0 & 1 & 0 & 0 & 0 & 1 \\
\hline P464 & CC-TG & 136 & FOXT_09879 & $\begin{array}{l}\text { hypothetical protein } \\
\text { similar to neutral } \\
\text { amino acid permease }\end{array}$ & $8 \mathrm{E}-40$ & transport & 0 & 0 & 0 & 0 & 0 & 0 & 0 & 2 & 0 & 0 & 0 & 1 \\
\hline P611 & $C T-G C$ & 284 & FOXG_09522 & $\begin{array}{l}\text { hypothetical protein } \\
\text { similar to } \\
\text { metalloreductase }\end{array}$ & $1 \mathrm{E}-139$ & transport & 0 & 0 & 0 & 0 & 0 & 0 & 0 & 3 & 0 & 0 & 0 & 3 \\
\hline P1293 & TC-CA & 396 & FVET_08954 & $\begin{array}{l}\text { hypothetical protein } \\
\text { similar to vacuolar } \\
\text { sorting protein } 35\end{array}$ & 0 & transport & 0 & 0 & 0 & 0 & 0 & 0 & 0 & 1 & 0 & 0 & 0 & 1 \\
\hline P365 & $\begin{array}{l}\text { CG- } \\
C G\end{array}$ & 426 & FOXT_12915 & arginase & 0 & virulence & 0 & 0 & 0 & 0 & 0 & 0 & 0 & 1 & 0 & 0 & 0 & 1 \\
\hline P610 & $C T-G C$ & 291 & FOXT_05960 & $\begin{array}{l}\text { hypothetical protein } \\
\text { similar to } \\
\text { peroxisomal PEX11 }\end{array}$ & $1 \mathrm{E}-136$ & virulence & 0 & 0 & 0 & 0 & 0 & 0 & 0 & 2 & 0 & 0 & 0 & \\
\hline
\end{tabular}

Selected FOM transcripts identified in infected melon plants, either specifically in planta, or both in planta and in vitro. Identification numbers (ID) correspond to progressive numbering of bands in the gels. The primer combination used to visualize each band (Bst-Mse column) and the corresponding length of the fragment are reported, along with the accession number in the database from which the sequence was retrieved, its annotation, the corresponding blast score and the functional category to which each transcript was assigned. In addition, the table reports the expression profile of each transcript, in each interaction and at each time point, estimated according to band intensity with a score from 1 to 3 .

\section{Analysis of $F$. oxysporum f. sp. melonis colonization in melon stems}

Because few researchers have investigated FOM infections in melon, the site and timing of recognition is currently unknown, which makes difficult to propose suitable time points for molecular analysis. We therefore began this investigation by characterizing the infection process in melon plants inoculated with avirulent FOM race 1 and virulent race 1,2. Disease progression was monitored using the same approach (plating stem fragments at different time points) that has been successful in tomato [49]. Colonization followed a similar trend to that reported for $F$. oxysporum f. sp. lycopersici in tomato [49], i.e. the fungus distribution was discontinuous in all combinations from 2-8 dpi, then continuous from 14-21 dpi with distinct patterns in the incompatible and compatible combinations. From $14 \mathrm{dpi}$ onwards, symptoms became 
Table 3 Real time RT-PCR expression analysis of selected melon genes

\begin{tabular}{|c|c|c|c|c|c|c|c|}
\hline ID & Homology & E-value & Annotation & ISPaVe strain/dpi & I/R & Fold-change (Infect. vs. mock) & SD \\
\hline P200 & MU48195 & 0 & ACC oxidase & 1070/4 dpi & I & 7.04 & 2.80 \\
\hline P557 & MU51460 & $3 E-45$ & Respiratory burst oxidase homolog protein $C$ & 1070/4 dpi & । & 61.92 & 14.73 \\
\hline P1100 & MU54228 & $2 \mathrm{E}-44$ & Protein translocase SEC61 & 1018/2 dpi & $\mathrm{R}$ & 1.87 & 0.37 \\
\hline P1370 & MU50486 & $1 \mathrm{E}-112$ & calcium-dependent protein kinase CDPK1444 & 1070/4 dpi & । & 3.81 & 0.66 \\
\hline P554 & MU45886 & $2 \mathrm{E}-27$ & 135-lipoxygenase & 1018/21 dpi & । & 1.26 & 0.14 \\
\hline P767 & MU47701 & $4 \mathrm{E}-50$ & Endochitinase 1 precursor & 1018/21 dpi & । & 473.05 & 146.45 \\
\hline P769 & MU43951 & $6 \mathrm{E}-61$ & UBQ14 (ubiquitin 14) & 1018/21 dpi & I & 25.34 & 24.29 \\
\hline
\end{tabular}

Seven melon transcripts, differentially expressed following Fusarium oxysporum f. sp. melonis (FOM) infection according to cDNA-AFLP experiments, were selected for validation by real time RT-PCR. Each gene was validated at the same time point and with the same FOM strain that generated a differential CDNA-AFLP profile. The FOM strains are ISPaVe170 race1 and ISPaVe1018 race 1,2. Results are shown as fold change values in infected samples in comparison to mockinoculated controls and are the average of three technical replicates. I/R: induced or repressed according to CDNA-AFLP experiments. SD: standard deviation.

obvious in the compatible interaction as generally reported in the literature $[49,11,20]$. Whereas the two virulent strains fully colonize the stem, colonization by the avirulent strain is reduced, and at 18 and $21 \mathrm{dpi}$ the height reached in stems is significantly lower than that reached at 2 and $4 \mathrm{dpi}$. These findings suggest that the plant may attack the invading pathogen and reduce its vitality. The data were confirmed by realtime PCR, indicating a progressive reduction in the amount of fungus present at later time points in the incompatible interaction (data not shown). Di Pietro and colleagues [2] found that, having reached the xylem, the fungus remains exclusively within the vessels using them to colonize the host rapidly, mainly through the production of microconidia rather than mycelia which, in turn, progressively grows inside the xylem inducing vessel clogging. In contrast to this prominent microconidia model $[20,23]$, studies using GFPlabeled $F$. oxysporum have shown that neither conidiophores nor microconidia are found in Arabidopsis or tomato xylem [21,22]. The response to infection may be affected by inoculum concentration, the age of the plant, the duration of exposure to the inoculum, and the type of substrate for plant growth (e. g. sand or soil) $[11,27]$. The assessment time points may also play an important role in the picture that emerges of the host/pathogen genetic responses. Nevertheless, differences in the infection process are likely to occur among different formae speciales and between different experimental designs.
Genetic elements of host colonization and pathogenicity Most transcriptomics studies involving F. oxysporum have focused on the interactions that occur in the xylem, and these studies suggest that the main resistance responses occur within or along the vessels. In this context, genes that are expressed solely in planta and not in artificial culture are the most interesting because they are likely virulence factors (Table 2 and Additional File 2). We identified 195 genes that were expressed in planta, 72 of which ( 37\%) were not expressed under artificial culture conditions and therefore represent putative virulence factors. Interestingly, only 11 out of 218 genes in cotton plants infected with F. oxysporum f. sp. vasinfectum were expressed specifically in planta [50]. The group of putative virulence factors identified in our analysis included plant cell wall degrading enzymes (CWDEs), represented by five transcripts encoding pectate lyases, endo- 1,4 beta xylanases and endo-1,4 beta glucanases, possibly activated by interaction with the host. Among these transcripts, an endo-1,4-beta-xylanase 2 precursor is the only sequence peculiar to race 1 , induced in the incompatible interaction, while the other four TDFs are specific to the race 1,2 strains. Like most fungi, $F$. oxysporum secretes CWDEs during either penetration or colonization [23]. Although the inactivation of individual CWDE- or protease-encoding genes might not have a detectable impact on virulence (reviewed in [2]), possibly because of functional redundancy, their activity is crucial in the process of fungal colonization. Active fungal growth is

Table 4 Real time RT-PCR expression analysis of selected Fusarium oxysporum f. sp. melonis (FOM) genes

\begin{tabular}{ccclcc}
\hline ID & Homology & E-value & Annotation & $\Delta$ Ct & SD \\
\hline P493 & FOXT_12671 & 5E-152 & Fusarium oxysporum f.sp lycopersici aspartate aminotransferase & 3.43 & 0.11 \\
P135 & FOXT_08523 & 1E-48 & Fusarium oxysporum f.sp lycopersici acyl-CoA desaturase & 16.11 & 0.41 \\
P1315 & FOXG_05190 & 2E-79 & Fusarium oxysporum f.sp lycopersici E3 ubiquitin-protein ligase & 3.99 & 0.31 \\
P819 & FOXG_09125 & 2E-117 & Fusarium oxysporum f.sp lycopersici hypothetical protein similar to avenacinase & 10.24 & 0.04 \\
P1403 & FOXT_13204 & 7E-51 & Fusarium oxysporum f.sp lycopersici predicted protein & 7.06 & 0.77 \\
\hline
\end{tabular}

Five Fusarium oxysporum f. sp. melonis transcripts, expressed in planta during infection according to cDNA-AFLP experiments, were selected for validation by Real time RT-PCR. Data are shown as the difference between the Ct of the gene analyzed and the Ct of melon actin (AY859055), used as a normalizer. 
also documented by the specific in planta expression of several genes related to carbohydrate and lipid metabolism, among them a squalene synthase involved in sterol biosynthesis. Sterols facilitate normal membrane function by controlling their fluidity, but they have also been implicated as ligands for nuclear receptors directly affecting transcription and signal transduction pathways [51]. Other examples include genes for cytoskeleton components (hypothetical protein similar to actin binding protein and dynamin-2) and a chitin synthase gene. Class V chitin synthase is a pathogenicity determinant in $F$. oxysporum and a mediator of protection against plant defense compounds [26].

Three other in planta-specific TDFs seem particularly important in terms of virulence. These represent genes encoding homologs of an avenacinase, a fumonisin 16p, and a siderophore iron transporter (mirB). There is increasing evidence that mycotoxin production may enhance pathogen virulence, especially fumonisins and some trichothecenes [52]. Fumonisin enhances the ability of $F$. graminearum to cause wheat head blight, one of the most important wheat diseases in the world [53]. It has been reported that mycotoxin production can be induced in fungi following the perception of the oxidative burst produced by the plant in response to infection, and could enhance pathogenicity by reducing the oxidative status of the fungal cell. Interestingly, the gene encoding the fungal toxin fumonisin was strongly and specifically expressed in planta only by virulent strains.

Avenacina is a hydrolytic enzyme that can degrade the oat saponin avenacine, and was first recognized as an essential pathogenicity factor in the take-all fungus Gaeumannomyces graminis var. avenae. Saponins, glycosides with soap-like properties that disrupt membranes, are a class of phytoanticipins. The role of saponin detoxification remains controversial in other plant-pathogen interactions $[54,55]$. However, the saponin-degrading tomatinase from $F$. oxysporum $\mathrm{f}$. sp. lycopersici has recently been confirmed as a virulence factor in tomato, by targeted disruption and overexpression of the corresponding gene [56]. In melon, we found that the avenacinase transcript is not only expressed specifically in planta, but is also differentially expressed between the two 1,2 strains, with higher levels produced by ISPaVe1018. To our knowledge, this is the first evidence to support a role for saponin-detoxifying enzymes in FOM infection. The siderophore iron transporter mir $\mathrm{B}$ gene may also represent a virulence factor because siderophores (lowmolecular-mass iron chelators that facilitate iron uptake and storage) are crucial for fungal pathogenicity in both animals and plants, and also maintain plantfungal symbioses [57]. The final group of FOM genes expressed only in planta includes several involved in transport and intracellular trafficking, and three related to signal transduction, with similarity to a calnexin involved in calcium-regulated protein folding [58], a phosphoserine phosphatase and a MADS box protein.

Although expressed both in planta and in vitro, a peroxisomal biogenesis factor PEX11 and an arginase coding gene are also worth mentioning (Table 2). Peroxisomes are single-membrane-bound organelles which, in filamentous fungi, are involved in the $\beta$-oxidation of fatty acids, peroxide detoxification and the occlusion of septal pores [27]. Peroxisomal function and fatty acid metabolism are required for fungal virulence. In $F$. oxysporum, four different Pex genes (PEX1, PEX10, PEX12 and PEX26) were identified as potential pathogenicity genes in a recent insertional mutagenesis screen, and the requirement for full pathogenicity was verified for two of them (PEX12 and PEX26) by complementation with the intact genes [59]. Arginase regulates the production of nitric oxide (NO), which is induced in a jasmonatedependent manner in response to wounding and is strongly implicated in the activation of disease resistance genes $[60,31]$. In microorganisms, arginase activity has been correlated with pathogenicity [61] and was shown to act as a bacterial survival mechanism by downregulating host nitric oxide production [62]. Other transcripts expressed by FOM in planta, specifically or otherwise, are involved in ubiquitinylation and protein degradation, both of which are necessary for pathogenicity in $F$. oxysporum f. sp. lycopersici [27], and in different aspects of fungal metabolism.

\section{Differentially expressed genes among $F$. oxysporum f. sp. melonis strains in vitro}

One major problem in FOM diagnosis is the identification of isolates at the race level. In melon-growing areas, the introduction of races that can overcome resistance in cultivated genotypes may result in dramatic crop losses. At present, no molecular tools are available to replace the time-consuming race-determination tests. We identified a number of transcripts with differential expression profiles between the two races (Additional File 4). Although differences in gene expression cannot be used directly as genetic markers of race identity, TDFs could be used as 'fingerprints' for this purpose. In addition, the differential virulence of the two 1,2 strains demonstrated by the host colonization pattern, could also be fingerprinted using TDFs that are differentially expressed between ISPaVe1018 and ISPaVe1083. Unfortunately, most TDFs in this category either matched hypothetical protein sequences in public databases or did not generate hits at all, and therefore do not allow speculation about the possible metabolic differences between the two races or between the two strains of FOM race 1,2 . 


\section{Large-scale transcriptional changes underlie disease development}

Transcriptional changes associated with resistance responses occur within the first $2 \mathrm{dpi}$, and are maintained with few changes thereafter (Figure 4). However, only 11 melon transcripts (Cluster A) are specific for the incompatible interaction. The largest group of modulated genes (Cluster D) is expressed in a non-specific manner, with variable modulation throughout the experiment, in both the incompatible and compatible interactions. The establishment of compatibility is characterized by a slightly delayed but progressive increase of the number of genes involved, underlying the significant metabolic disturbances that might be associated with symptom development. The majority of these changes are included in Cluster D and are thus non-specific up to $8 \mathrm{dpi}$, but are followed by a sudden wave of susceptibility-specific transcriptional changes at $21 \mathrm{dpi}$, almost completely conserved between the virulent strains ISPaVe1018 and ISPaVe1083.

Although the precocity of the resistance response is expected, the small number of genes involved is unexpected. Incompatible interactions commonly involve large-scale transcriptional reprogramming toward defense, which is generally more intense and rapid than in corresponding compatible interactions $[63,64,28]$. However, vascular diseases may represent a peculiar situation, in which symptom development and consequent damage could depend not only on the pathogenetic activity of the fungus but also the strength and timing of the host response. This was indicated by pioneering research in which delayed formation of tyloses in susceptible genotypes eventually contributes to vessel clogging [23]. In agreement with the above, our data suggest that more striking changes in gene expression accompany disease and symptom development than resistance, thus resistance might depend more on the ability to tolerate the infection, avoiding reactions.

\section{Transcriptional changes in the compatible interaction}

Although cluster analysis and functional annotation of the identified melon transcripts provided an overview of the transcriptional changes occurring in infected melon plants, the limited availability of sequence data made it difficult to draw firm conclusions about the molecular events occurring in infected plants. Of the 115 TDFs identified in Cluster C, 41 did not match any database sequences or matched sequences that have yet to be annotated. The remaining 74 TDFs encode well-known components of disease resistance responses and related signal transduction cascades, such as calmodulin and calmodulin-binding proteins, transcription factors, a 12oxophytodienoate reductase, and a 13S-lipoxygenase involved in jasmonic acid biosynthesis [65], and enzymes involved in the biosynthesis of secondary metabolites acting as antimicrobial compounds, or in a general stress responses, such as xanthine dehydrogenase and betaine aldehyde dehydrogenase [66,67]. Genes encoding pathogenesis-related proteins such as endochitinase, beta-1,3 glucanase and a type I proteinase inhibitor-like protein were also specifically modulated in the compatible interaction. Altogether, transcripts related to the defense, response to stimulus and secondary metabolism categories accounted for $\sim 25 \%$ of modulated TDFs in Cluster $\mathrm{C}$. These findings further support the hypothesis that a delayed defense response might indeed be responsible for symptom development.

Cluster $\mathrm{C}$ also contained genes potentially involved in the establishment of susceptibility, such as those related to auxin accumulation. Several reports indicate that an increase in auxin levels in the cell can contribute to disease susceptibility [68] and that a similar increase can be induced by pathogens in order to facilitate colonization. TDFs with homology to an indole-3-acetic acidamino synthetase and to an IAA-type protein Q75GK0 [68] are specifically induced in the compatible interaction. However, other genes in Cluster D that induce auxin signaling (e.g. auxin response factor 6 and AUX1like auxin transport protein) are repressed by both virulent strains, but induced by the avirulent strain at 21 dpi. The overall picture is therefore complex and suggests that the compatible interaction mainly involves transcriptional changes that are otherwise typical of effective resistance responses. It is tempting to speculate that the recessive resistance identified in Asian accessions might be related to the lack of a plant reaction and thus to better tolerance of the infection process [20].

\section{Transcriptional changes in the incompatible interaction}

Resistance responses are generally characterized by rapid and extensive reprogramming of transcriptional activity, especially in race-specific interactions. However, that resistance of Charentais Fom-2 to FOM race 1, although mediated by a single R-type resistance gene, is not complete, since the fungus can always be reisolated from the stem of Charentais Fom-2 plants. In our model system we noted surprisingly few transcriptional changes specifically associated with the incompatible interaction (11 TDFs, Cluster A). These included a calmodulin-related protein, stably upregulated from 4 dpi onwards, which plays an important role in the transduction of calcium signaling and could be involved in the resistant response (as already demonstrated in other species [69]) and a catalase that may protect the pathogen from the strong oxidative burst associated with resistance.

Additional information about the resistance response could be found by analyzing genes that are modulated 
in both interactions (Cluster D) but with a peculiar pattern (e.g. earlier or more strongly in the incompatible interaction), or those showing a reverse modulation of gene expression in one or the other interactions (which might reveal specific requirement in each interaction). An interesting finding is that many of the genes modulated earlier or more strongly during resistance are classified as signal transduction-related genes (Additional File 1). These include several transcripts involved in calcium signaling (calcium-transporting ATPase, calcium dependent protein kinase and calcium-binding protein), transcription factors, kinases and a homolog of NDR1 (non race-specific disease resistance 1). This last gene is induced at 2 and 4 dpi following infection with race 1 and only at $8 \mathrm{dpi}$ following infection with race 1,2 . NDR1 was originally identified in Arabidopsis as a factor required for resistance to both bacterial and fungal pathogens [70] and it is known to mediate resistance controlled by $\mathrm{R}$ genes of the nucleotide binding site leucine rich repeats (NBS-LRR) class, which is distinct from the Toll/interleukin receptor (TIR) class [71]. Fom2 in Charentais Fom-2 plants is indeed a non-TIR $R$ gene, although with a peculiar structure that lacks the typical N-terminal coiled-coil domain [72]. Therefore, it seems plausible that its action might require NDR1.

Other genes modulated earlier in the establishment of resistance include two adenosylhomocysteinases, an aspartokinase-homoserine dehydrogenase and a serine carboxypeptidase. An Arabidopsis adenosylhomocysteinase encoded by the gene HOG1 (homology dependent gene silencing 1) is required for DNA-methylation gene silencing [73]. The involvement of RNA-silencing machinery in plant innate immunity has recently been demonstrated not only against viruses but also bacterial and fungal pathogens, including Verticillium in Arabidopsis $[74,75]$. The same transcript increases in aphidinfested sorghum plants [76]. The potential involvement of these candidate genes in Charentais Fom-2- controlled resistance could be the object of future investigations.

Most genes in Cluster D are not differentially modulated in the incompatible and compatible interactions. Interesting examples include TDFs related to ACC oxidases. These enzymes participate in the last step of ethylene biosynthesis and are involved in the response to stress and to pathogens, but are also implicated in senescence, necrosis and disease development. Ethylene has been associated with both wilting and resistance against vascular diseases [77]. We detected four transcripts with similarity to ACC oxidases. These showed variable expression profiles, but there was no difference between compatible and incompatible interactions, which suggests that ethylene might be involved in both susceptibility and resistance. In melon, different ACC oxidase genes are induced differentially during development and pathogen infection [78]. The same variable modulation has been detected for other transcripts possibly related to jasmonate biosynthesis, such as allene oxide synthase and the 13S-lipoxygenase mentioned above.

\section{Conclusions}

In conclusion, our data suggest that resistance against FOM in melon involves only limited transcriptional changes, and that wilting symptoms could derive, at least partially, from an active plant response.

A small but important collection of FOM transcripts were shown to be expressed specifically in planta, and not in the same fungal strains growing in vitro, providing excellent candidate virulence factors which can be investigated further to learn more about the molecular basis of host-pathogen interactions in melon. Finally, race-specific genes were expressed in fungal colonies in vitro as well as in planta, suggesting they could be developed as markers in molecular race-determination assays that could replace the current laborious inoculation-based methods.

\section{Methods}

\section{Plant material}

Seeds from melon (Cucumis melo L.) genotype Charentais Fom-2 (resistant to races 0 and 1, and susceptible to race 1,2 ) were surface sterilized with $1 \% \mathrm{NaOCl}$ for 20 min and incubated in sterile distilled water at $4^{\circ} \mathrm{C}$ overnight. The seeds were pre-germinated on filter paper, and seedlings were cultivated in plastic pots filled with sterilized soil in the greenhouse at $25 \pm 2{ }^{\circ} \mathrm{C}$ with 80 $90 \%$ relative humidity.

\section{Pathogen material and production of the inoculum}

Virulent F. oxysporum f. sp. melonis Snyder \& Hans. (FOM) strains were obtained from the fungal collection of the Plant Pathology Research Center (CRA-PAV) [79]. Three strains were used as inoculum, namely ISPaVe1070 (race 1), and ISPaVe1018 and ISPaVe1083 (race 1,2). Race designation had been achieved by inoculation on different hosts according to the nomenclature proposed by Risser et al. [5]. Inoculums were produced by growing each strain on $90-\mathrm{mm}$ Petri dishes containing potato-dextrose agar (PDA, Oxoid). Fourteen-day-old cultures grown at $24^{\circ} \mathrm{C}$ were flooded with sterile distilled water and gently scraped with a sterile glass rod to obtain a spore suspension. This was filtered through two layers of cheesecloth and the filtrate was diluted to obtain the inoculum at a concentration of $1 \times 10^{6}$ conidia $/ \mathrm{ml}$.

\section{Inoculation procedure}

Charentais Fom-2 melon seedlings were inoculated at the four-to-five true leaf stage [18]. The roots of each seedling were gently washed in tap water, pruned by 
approximately $1 \mathrm{~cm}$ and dipped for $30 \mathrm{~min}$ in the conidial suspension. Control seedlings were dipped in sterile distilled water. Seedlings were then transferred into plastic pots filled with sterilized soil and maintained in the greenhouse at $25 \pm 2{ }^{\circ} \mathrm{C}$ with $80-90 \%$ relative humidity. For each fungal strain, a total of 72 plants (eight reisolation times after inoculation multiplied by nine replicates) was used to investigate vascular colonization, and 20 plants (four plants per each time point) were used for RNA extraction and transcriptomic analysis.

\section{Vascular colonization}

After inoculation, seedlings were monitored for fungal colonization along the stem by reisolation. The experiment was concluded at $21 \mathrm{dpi}$, when all plants undergoing the compatible interaction (Charentais Fom-2 inoculated with race 1,2) displayed obvious and severe wilting symptoms. Nine plants for each strain were cut at the stem base 1, 2, 4, 8, 14, 16, 18 and $21 \mathrm{dpi}$, and were defoliated. After discarding the basal $15 \mathrm{~mm}$, the stems were cut into 12 sections, each $5 \mathrm{~mm}$ in length, to a maximum height of $75 \mathrm{~mm}$. Sections were placed on PDA, incubated at room temperature for 8 days and examined every day for the appearance of fungal outgrowths. A completely randomized distribution was adopted for the melon plants kept in the greenhouse as well as for the Petri-dishes with stem sections incubated in the laboratory.

\section{Data analysis}

Vascular colonization was scored according to the frequency of successful reisolation in stem sections arranged in four height classes measured from the stem base: $15-30 \mathrm{~mm}, 30-45 \mathrm{~mm}, 45-60 \mathrm{~mm}$ and $60-75 \mathrm{~mm}$. Percentage values grouped in the four height classes were subjected to a two-way ANOVA for each height class and for the total of the four classes. The two factors considered were strain and time (dpi). The data did not match the parametric ANOVA requirements (normal data distribution and homogeneous variance) with any transformation, so the non-parametric Monte Carlo permutation test was used instead. The probabilities of the main effects of each factor were generated by restricting permutations within the levels of the other factor [80], whereas the interaction between strain and time was tested by unrestricted permutations after the calculation of residuals [81]. The statistical test used for the main factors was the sum of squares between groups $\left(Q_{b}\right)$, whereas the test used for interaction was the pseudo F-ratio $\left(F=Q_{b} / Q_{w}\right.$, where $Q_{w}$ is the sum of squares within groups). Because of interactions between factors present in all five two-way ANOVA tests, the effect of time was tested separately for each strain in a one-way ANOVA either for each height class or for the total of the four classes. Follow-up tests of the differences between times were performed by considering all possible pairwise contrasts. In this case, to avoid inflation of the type I error rate, a Bonferroni-corrected significance level of $\mathrm{P} \leq 0.0018$ ( $\mathrm{P}<0.05$ divided by 28 , which is the total number of possible contrasts between the eight reisolation times) was calculated and used as minimum nominal $\mathrm{P}$-value to obtain an actual $\mathrm{P} \leq 0.05$ value. The statistical results refer to the analysis performed on the total of the four height classes for each strain.

To characterize the continuity of the distribution of the fungus along the stem, a continuity index was calculated based on the reisolation data. The index was determined for each plant by considering the presence or absence of the fungus in the pairs of subsequent stem sections and assigning a value of 1 when the fungus was reisolated or not reisolated in both sections and a value of 0 when it was reisolated only in one of the two sections. The index was then calculated by averaging the obtained values.

\section{RNA extraction procedure}

For each plant tissue sample, $\sim 2$ g of stem segments (four plants) were excised with a sterile razor blade, dehydrated in liquid nitrogen and stored at $-80^{\circ} \mathrm{C}$. Total RNA was extracted using TRIzol reagent (Invitrogen) and treated with DNase (Sigma-Aldrich) following the manufacturer's instructions. For fungal colonies, total RNA was extracted from frozen single-spore colonies (60-150 mg), grown for 8 days on PDA at $24^{\circ} \mathrm{C}$, with the RNeasy Mini kit (Qiagen) following the manufacturer's protocol for plant tissues.

\section{cDNA-AFLP analysis}

We used the cDNA-AFLP protocol described by Vos et al. [82] and Bachem et al. [83] with the modifications and primers described by Breyne et al. [84] which permit the visualization of a single cDNA fragment for each mRNA present in the original sample, reducing the output sequence redundancy. Double-stranded cDNA was synthesized from $2.5 \mu \mathrm{g}$ total RNA using the Superscript II reverse transcription kit (Invitrogen) and a biotinylated oligo-dT primer (Promega).

After pre-amplification, the mixture was diluted 600fold and $5 \mu \mathrm{l}$ was used for selective amplification with 128 primer combinations, carried out with one selective nucleotide added on the ${ }^{33} \mathrm{P}$-labeled Bst $\mathrm{YI}$ primer and two selective nucleotides on the MseI primer. We used the following touch-down PCR conditions: 2 min denaturation at $94^{\circ} \mathrm{C}$ followed by $30 \mathrm{~s}$ denaturation at $94^{\circ} \mathrm{C}$, $30 \mathrm{~s}$ annealing at $65^{\circ} \mathrm{C}, 60 \mathrm{~s}$ extension at $72^{\circ} \mathrm{C}$ for 13 cycles (scaledown of $0.7^{\circ} \mathrm{C}$ per cycle); $30 \mathrm{~s}$ denaturation at $94^{\circ} \mathrm{C}, 30 \mathrm{~s}$ annealing at $56^{\circ} \mathrm{C}, 60 \mathrm{~s}$ extension at $72^{\circ} \mathrm{C}$ 
for 23 cycles, and $5 \mathrm{~min}$ at $72^{\circ} \mathrm{C}$. Selective $\left[\gamma_{-}{ }^{33} \mathrm{P}\right] \mathrm{ATP}-$ labeled amplification products were separated on a $6 \%$ polyacrylamide gel in a Sequi-Gen GT Sequencing Cell $(38 \times 50 \mathrm{~cm})($ Bio-Rad) running for $2.5 \mathrm{~h}$ at $115 \mathrm{~W}$ and $50^{\circ} \mathrm{C}$. Gels were dried onto $3 \mathrm{MM}$ Whatman paper for 2 $\mathrm{h}$ at $80^{\circ} \mathrm{C}$ on a Gel Dryer Model 583 (Bio-Rad) and marked with Glogos II Autorad Markers (Stratagene) before exposing to Kodak Biomax MR films, for 12-16 h.

\section{Sequence analysis of cDNA-AFLP fragments}

Bands corresponding to differentially expressed genes were excised from the gels with a surgical blade and the eluted DNA was reamplified using non-labeled primers identical to those employed for selective amplification and the following PCR conditions: 15 min denaturation at $94^{\circ} \mathrm{C}, 40 \mathrm{~s}$ denaturation at $94^{\circ} \mathrm{C}, 60 \mathrm{~s}$ annealing at $56^{\circ}$ C, $40 \mathrm{~s}$ extension at $72^{\circ} \mathrm{C}$ for 35 cycles, and $5 \mathrm{~min}$ at $72^{\circ}$ C. The quantity of each reamplified bands were checked on a $1.8 \%$ agarose gel against the 1650-bp fragment of the DNA ladder $1 \mathrm{~Kb}$ plus (Invitrogen). PCR products were purified with MultiScreen PCR $\mu 96$ plates (Millipore) and sequenced directly (BMR Genomics). Sequence information was obtained by comparing nucleotide and protein sequences in the available public databases by BLAST sequence alignment [85]. Homology searching was carried out against the following databases: NCBI [34], Cucurbit Genomic Database Melon Unigene ver. 4.0 [32], UNIPROT database [33] and Fusarium Comparative Database [39]. Sequences were manually assigned to functional categories based on the analysis of scientific literature and also with the aid of the information reported for each sequences by the Gene Ontology Consortium [35], where available.

Sequence data from this article have been deposited in GenBank, Accession Numbers: HO867279- HO867981.

\section{Real-time RT-PCR analysis}

Real-time RT-PCR was carried out on pools of RNA derived from two independent biological experiments. All samples were examined as three technical replicates. Samples were prepared from whole stems of infected and mock-inoculated plants (T0) corresponding, for each gene tested, to the same interaction type and time point that indicated differential expression in the equivalent cDNA-AFLP experiment. The FOM strains were ISPaVe170 (race1) and ISPaVe1018 (race $1,2 \mathrm{w}$ ). Total RNA was treated with RNase-free DNase (Sigma-Aldrich) according to the manufacturer's instructions, and $3 \mu \mathrm{g}$ was then used for reverse transcription on Ready-To-Go you-prime first-strand beads (GE Healthcare). Then $5 \mu \mathrm{l}$ of 1:10 diluted cDNA samples was used as the qRT PCR template in a $25-\mu \mathrm{l}$ total volume containing $0.4 \mu \mathrm{M}$ gene-specific primers and $12.5 \mu$ platinum SYBR Green qPCR SuperMix with ROX (Invitrogen). All samples were examined in three technical replicates. Experiments were carried out in a Mx3000P QPCR Systems (Stratagene) with the following thermal cycling profile: $95^{\circ} \mathrm{C}$ for $10 \mathrm{~min} ; 40$ cycles of $95^{\circ} \mathrm{C}$ for $30 \mathrm{~s}, 55^{\circ} \mathrm{C}$ for $30 \mathrm{~s}, 72^{\circ} \mathrm{C}$ for $30 \mathrm{~s}$. Each real-time assay was tested in a dissociation protocol to ensure that each amplicon was a single product. Relative quantification of gene expression was performed using the housekeeping gene actin [31]. The actual stability of actin expression was tested in preliminary experiments, calculating the coefficient of variation $(\mathrm{CV}=0.047)$ of the threshold cycle for actin amplification in all infection conditions and in mock inoculated controls [86]. The primers were designed according to the melon actin sequence in GenBank (AY859055): forward, 5'-CCC TGG TAT TGC AGA CAG GA-3' and reverse, 5'-ACA TCT GCT GGA AGG TGC TT-3'. A control experiment without cDNA was included for each PCR mix. Specific primer pairs (20 bp) were designed for the 12 TDFs chosen for validation, using the Primer 3 software (Additional File 5). Data were analyzed using MxPro QPCR software (Stratagene). The $C_{t}$ (cycle at which the increase of fluorescence exceeded the threshold setting) was used to calculate the fold changes (FC) in each infected sample compared to the expression level detected in the corresponding sample under control conditions (baseline) with the following formula: $\mathrm{FC}=$ $2^{-\Delta \Delta C t}$ where $\Delta \Delta C_{t}=\left(C_{t}\right.$ target $-C_{t}$ actin $)$ infected sample $\left(C_{t}\right.$ target $-C_{t}$ actin $)$ uninfected sample.

Evaluation of expression of FOM genes was carried out by calculating the difference between the $\mathrm{Ct}$ of the gene analyzed and the Ct of melon actin (AY859055), used as a normalizer.

\section{Additional material}

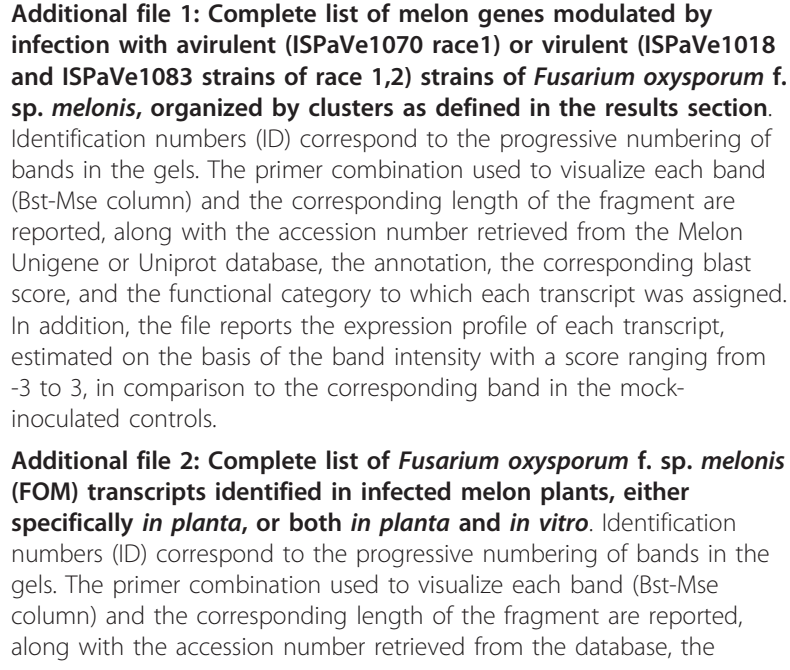

Additional file 2: Complete list of Fusarium oxysporum f. sp. melonis (FOM) transcripts identified in infected melon plants, either specifically in planta, or both in planta and in vitro. Identification numbers (ID) correspond to the progressive numbering of bands in the gels. The primer combination used to visualize each band (Bst-Mse column) and the corresponding length of the fragment are reported, along with the accession number retrieved from the database, the 
annotation, the corresponding blast score, and the functional category to which each transcript was assigned. In addition, the file reports the expression profile of each transcript, in each interaction and at each time point, estimated on the basis of the band intensity with a score ranging from 1 to 3.

Additional file 3: List of the core $\mathbf{5 4}$ melon genes, which remain modulated in a coherent way (induced or repressed) throughout the experiment (from $4 \mathrm{dpi}$ onwards) in the incompatible interaction between melon and the FOM race 1 strain ISPaVe1070.

Additional file 4: Complete list of Fusarium oxysporum f. sp. melonis (FOM) transcripts identified by CDNA-AFLP analysis from fungal samples of colonies grown in vitro and selected for sequencing. TDFs were chosen on the basis of their differential abundance in the different FOM strains. Identification numbers (ID) correspond to the progressive numbering of bands in the gels. The primer combination used to visualize each band (Bst-Mse column) and the corresponding length of the fragment are reported, along with the accession number retrieved from the database and the corresponding blast score and annotation. In addition, the file reports the presence (+) or absence (-) of expression of each transcript for the 3 strains of FOM.

Additional file 5: List of primers used in Real time RT-PCR analysis. Identification numbers (ID) correspond to the progressive numbering of bands in the gels. The accession codes for each gene represent the corresponding database in which homology was identified, $\mathrm{MU}=$ Melon Unigene, FOX = Fusarium Comparative Database.

\section{Acknowledgements}

This research was financially supported by the project FUSAMELO " Indagini sui meccanismi di virulenza/resistenza alla Fusariosi per la selezione e costituzione di varietà resistenti di melone" granted by the Italian Ministry of Agricultural, Alimentary and Forest Politics (MiPAAF).

\section{Author details}

${ }^{1}$ Agricultural Research Council (CRA), Research Unit for Vegetable Crop in Central Areas, Via Salaria 1, 63030 Monsampolo del Tronto (AP), Italy. ${ }^{2}$ Department of Biotechnology, University of Verona, Strada Le Grazie 15, 37134 Verona, Italy. ${ }^{3}$ Agricultural Research Council (CRA), Plant Pathology Research Center, Via C.G. Bertero 22, 00156 Roma, Italy. ${ }^{4}$ Department of Environmental Agronomy and Crop Production, University of Padova, Viale dell'Università 16, 35020 Legnaro, Padova, Italy.

\section{Authors' contributions}

SS participated in the scoring of the c DNA-AFLP bands, performed blast and database homology search and the assignment of the functional categories. The author performed the real time experiments and collaborated in writing the manuscript and in preparing table and figures. AP participated in interpretation and discussion of CDNA-AFLP data and wrote most of the manuscript in particular all parts related to plant responses to infections. The author also collaborated in preparing table and figures. LL performed the infections and the reisolations from inoculated plants, collected and processed infection data, performed the extraction of RNA from fungal colonies, and checked the autoradiographic films for profile clustering. AF performed all blast and database homology searches and profile clustering. MS performed the statistical analysis and the description of the different patterns of the host colonization by the three pathogenic strains of FOM. MD participated in conceiving the study and to produce the CDNA-AFLP data. $J H$ performed DNA and RNA extraction from melon plants, participated in CDNA-AFLP and in real-time RT-PCR experiments. NF prepared and provided all the genetic material used in this work and participated in the interpretation and discussion of CDNA-AFLP data. $A B$ is the research coordinator, conceived the study, participated in all steps of the analysis and extensively collaborated in writing the manuscript, in particular on aspects related to fungal infection process, vascular colonization and molecular basis of pathogenicity. All authors read and approved the final manuscript.

Received: 25 October 2010 Accepted: 21 February 2011

Published: 21 February 2011

\section{References}

1. Armstrong GM, Armstrong JK: Formae speciales and races of Fusarium oxysporum causing wilt disease. In Fusarium: disease, biology, and taxonomy. Edited by: Nelson PE, Toussoun TA, Cook RJ. University Park, PA: Pennsylvania State University Press; 1981:391-399.

2. Di Pietro A, Madrid MP, Caracuel Z, Delgado-Jarana J, Roncero MIG: Fusarium oxysporum: exploring the molecular arsenal of a vascular wilt fungus. Molecular Plant Pathology 2003, 4:315-325.

3. Gordon TR, Martyn RD: The evolutionary biology of Fusarium oxysporum. Ann Rev Phytopathol 1997, 35:11-128.

4. Suarez-Estrella F, Vargas-Gracia MC, Lopez MJ, Moreno J: Survival of Fusarium oxysporum f. sp. melonis on plant waste. Crop Protection 2004, 23:127-133.

5. Risser G, Banihashemi Z, Davis DW: A proposed nomenclature of Fusarium oxysporum f. sp. melonis races and resistance genes in Cucumis melo. Phytopathology 1976, 66:1105-1106.

6. Messianen CM, Risser G, Pecaut P: Etude des plantes resistantes au Fusarium oxysporum f. sp. melonis dans la variété de melon Cantaloup Charentais. Ann Amélio Plantes 1962, 12:157-164.

7. Zink FW, Gubler WD: Inheritance of resistance in muskmelon to Fusarium wilt. J Am Soc Hort Sci 1985, 110:600-604.

8. Perchepied L, Pitrat M: Polygenic inheritance of partial resistance to Fusarium oxysporum f.sp. melonis race 1,2 in melon. Phytopathology 2004, 94:1331-1336.

9. Ficcadenti N, Sestili S, Annibali S, Campanelli G, Belisario A, Maccaroni M, Corazza L: Resistance to Fusarium oxysporum f.sp. melonis race 1,2 in muskmelon lines Nad-1 and Nad-2. Plant Dis 2002, 86:897-900.

10. Perchepied L, Dogimont C, Pitrat M: Strain-specific and recessive QTLs involved in the control of partial resistance to Fusarium oxysporum f.sp. melonis race 1,2 in a recombinant inbred line population in melon. Theor Appl Genet 2005, 111:65-74

11. Herman R, Perl-Traves R: Characterization and inheritance of a new source of resistance to Fusarium oxysporum f. sp. melonis race 1,2 in Cucumis melo. Plant Dis 2007, 91:1180-1186.

12. Herman R, Zvirin Z, Kovalski I, Freeman S, Denisov Y, Zuri G, Katzir N, PerlTraves $R$ : Characterization of Fusarium race 1,2 resistance in melon and mapping of major QTL for this trait near a fruit netting locus. Proceedings of the IX Eucarpia meeting on genetics and breeding of Cucurbitaceae Avignon (France); 2008.

13. Wechter WP, Whitehead MP, Thomas CE, Dean RA: Identification of a randomly amplified polymorphic DNA marker linked to the Fom-2 Fusarium wilt resistance gene in muskmelon MR-1. Phytopathology 1995, 85:1245-1249.

14. Belisario A, Corazza L: Contro la tracheofusariosi del melone c'è solo il miglioramento genetico. Colture Protette 2003, 2:41-43.

15. Gordon TR, Okamoto D, Jacobson DJ: Colonization of muskmelon and non susceptible crops by Fusarium oxysporum f. sp. melonis and other species of Fusarium. Phytopathology 1989, 79:1095-1100.

16. Lievens B, Rep M, Thomma BPHJ: Recent developments in the molecular discrimination of formae speciales of Fusarium oxysporum. Pest Manag Sci 2008, 64:781-788.

17. Di Pietro AD, Garcia-Maceira FI, Meglecz E, Roncero IG: A MAP kinase of the vascular wilt fungus Fusarium oxysporum is essential for root penetration and pathogenesis. Mol Microbiol 2001, 39:1140-1152.

18. Inoue I, Namiki F, Tsuge T: Plant colonization by vascular wilt fungus Fusarium oxysporum requires FOW1, a gene encoding a mitochondrial protein. The Plant Cell 2002, 14:1869-1883.

19. Lagopodi AL, Ram AFJ, Lamers GEM, Punt PJ, Van den Hondel CAMJJ, Lugtenberg BJJ, Bloemberg GV: Novel aspects of tomato root colonization and infection by Fusarium oxysporum f. sp. radicis-lycopersici revealed by confocal laser scanning microscopic analysis using the green fluorescent protein as a marker. Mol Plant Microbe Interact 2002, 15(2):172-179.

20. Zvirin T, Herman R, Brotman Y, Denisov Y, Belausov E, Freeman S, PerlTreves R: Differential colonization and defence responses of resistant and susceptible melon lines infected by Fusarium oxysporum race 1-2. Plant Pathol 2010, 59:576-585.

21. Czymmek KJ, Fogg M, Powell DH, Sweigard J, Park SY, Kang S: In vivo timelapse documentation using confocal and multi-photon microscopy reveals the mechanisms of invasion into the Arabidopsis root vascular system by Fusarium oxysporum. Fungal Genet Biol 2007, 44:1011-1023. 
22. Van der Does HC, Duyvesteijna RGE, Goltstein PM, van Schiea CCN, Mandersc EMM, Cornelissen BJC, Rep M: Expression of effector gene SIX1 of Fusarium oxysporum requires living plant cells. Fungal Genet Biol 2008, 45(9):1257-1264.

23. Beckman $\mathrm{CH}$ : The nature of wilt diseases of plants. St. Paul, MN: American Phytopathological Society; 1987.

24. Agrios GN: Plant Pathology. Academic Press, New York; 1997

25. Takken F, Rep M: The arms between tomato and Fusarium oxysporum. Mol Plant Pathol 2010, 11:309-314.

26. Madrid MP, Di Pietro A, Roncero Ml: Class V chitin synthase determines pathogenesis in the vascular wilt fungus Fusarium oxysporum and mediates resistance to plant defence compounds. Mol Microbiology 2003, 46:257-266.

27. Michielse $C B$, Rep M: Pathogen profile update: Fusarium oxysporum. Mol Plant Pathol 2009, 10:311-324.

28. Wise RP, Moscou MJ, Bogdanove AJ, Whitham SA: Transcript profiling in host-pathogen interactions. Annu Rev Phytopathol 2007, 45:329-369.

29. Roh SW, Abell GC, Kim KH, Nam YD, Bae JW: Comparing microarrays and next-generation sequencing technologies for microbial ecology research. Trends Biotechnol 2010, 28:291-299.

30. Vuylsteke M, Peleman JD, van Eijk MJT: AFLP-based transcript profiling (cDNA-AFLP) for genome-wide expression analysis. Nature Protocols 2007, 2:1399-1413.

31. Gupta S, Chakraborti D, Rangi RK, Basu D, Das S: A molecular insight into the early events of Chickpea (Cicer arietinum) and Fusarium oxysporum $\mathrm{f}$. sp. ciceri (Race 1) interaction through cDNA-AFLP analysis. Phytopathology 2009, 99:1245-1257.

32. Gonzalez-lbeas D, Blanca J, Roig C, González-To M, Picó B, Truniger V, Gómez P, Deleu W, Caño-Delgado A, Arús P, Nuez F, Garcia-Mas J, Puigdomènech $P$, Aranda MA: MELOGEN: an EST database for melon functional genomics. BMC Genomics 2007, 8:306.

33. Uniprot database. [http://www.uniprot.org/].

34. NCBI. [http://www.ncbi.nlm.nih.gov/].

35. The Gene Ontology Consortium: Gene Ontology: tool for the unification of biology. Nat Genet 2000, 25:25-29.

36. Kurkcuoglu S, Degenhardt J, Lensing J, Al-Masri AN, Gau AE: Identification of differentially expressed genes in Malus domestica after application of the non-pathogenic bacterium Pseudomonas fluorescens Bk3 to the phyllosphere. J Exp Bot 2007, 58(3):733-741.

37. Guo L, Chen S, Liu K, Liu Y, Ni L, Zhang K, Zhang L: Isolation of Heat Shock Factor HsfA1a-binding sites in vivo revealed variations of Heat Shock elements in Arabidopsis thaliana. Plant Cell Physiol 2008, 49(9):1306-1315.

38. Titiz O, Tambasco-Studart M, Warzych E, Appel K, Amrhein N, Laloi C, Fitzpatrick TB: PDX1 is essential for vitamin B6 biosynthesis, development and stress tolerance in Arabidopsis. The Plant Journal 2006, 48:933-946.

39. Fusarium Comparative Database. [http://www.broad.mit.edu/annotation/ genome/fusarium_group/MultiHome.html].

40. Czechowski T, Bari RP, Stitt M, Scheible WR, Udvardi MK: Real-time RT-PCR profiling of over 1400 Arabidopsis transcription factors: Unprecedented sensitivity reveals novel root- and shoot specific genes. Plant J 2004, 38:366-379.

41. Svensson JT, Crosatti C, Campoli C, Bassi R, Stanca AM, Close TJ, Cattivelli L: Transcriptome analysis of cold acclimation in barley Albina and Xantha mutants. Plant Physiol 2006, 141:257-270.

42. Nieto C, Morales M, Orjeda G, Clepet C, Monfort A, Sturbois B, Puigdomènech P, Pitrat M, Caboche M, Dogimont C, Garcia-Mas J, Aranda MA, Bendahmane A: An elF4E allele confers resistance to an uncapped and nonpolyadenylated RNA virus in melon. Plant J 2006, 48:452-462.

43. Mascarell-Creus A, Cañizares J, Vilarrasa-Blasi J, Mora-García S, Blanca J, Gonzalez-Ibeas D, Saladié M, Roig C, Deleu W, Picó-Silvent B, López-Bigas N, Aranda MA, Garcia-Mas J, Nuez F, Puigdomènech P, Caño-Delgado Al: An oligo-based microarray offers novel transcriptomic approaches for the analysis of pathogen resistance and fruit quality traits in melon (Cucumis melo L.). BMC Genomics 2009, 10:467.

44. Durrant WE, Rowland O, Piedras P, Hammond-Kosack KE, Jones JDG: cDNAAFLP reveals a striking overlap in race-specific resistance and wound response gene expression profiles. The Plant Cell 2000, 12:963-977.

45. Guo J, Jiang RHY, Kamphuis LG, Govers F: A cDNA-AFLP based strategy to identify transcripts associated with avirulence in Phytophthora infestans. Fungal Genet Biol 2006, 43(2):111-123.
46. Polesani M, Desario F, Ferrarini A, Zamboni A, Pezzotti M, Kortekamp A, Polverari A: c-DNA-AFLP analysis of plant and pathogen genes expressed in grapevine infected with Plasmopara viticola. BMC Genomics 2008, 9:142-153.

47. Wang X, Tang C, Zhang G, Li Y, Wang C, Liu B, Qu Z, Zhao J, Han Q, Huang L, Chen X, Kang Z: CDNA-AFLP analysis reveals differential gene expression in compatible interaction of wheat challenged with Puccinia striiformis f. sp. tritici. BMC Genomics 2009, 10:289.

48. Gupta S, Chakraborti D, Sengupta A, Basu D, Das S: Primary metabolism of chickpea is the initial target of wound inducing early sensed Fusarium oxysporum f. sp. ciceri Race 1. PLoS ONE 2010, 5(2):e9030.

49. Rodríguez-Molina MC, Medina I, Torres-Vila LM, Cuartero J: Vascular colonization patterns in susceptible and resistant tomato cultivars inoculated with Fusarium oxysporum f.sp. lycopersici races 0 and 1. Plant Pathology 2003, 52:199-203

50. McFadden HG, Wilson IW, Chapple RM, Dowd C: Fusarium wilt (Fusarium oxysporum f. sp. vasinfectum) genes expressed during infection of cotton (Gossypium hirsutum). Mol Plant Pathol 2006, 7:87-101.

51. Devarenne TP, Ghosh A, Chappell J: Regulation of squalene synthase, a key enzyme of sterol biosynthesis, in tobacco. Plant Physiol 2002, 129:1095-1106.

52. Reverberi M, Ricelli A, Zjalic S, Fabbri AA, Fanelli C: Natural functions of mycotoxins and control of their biosynthesis in fungi. J Appl Microbiol Biotechnol 2010, 87(3):899-911.

53. Alexander NJ, Proctor RH, McCormick SP: Genes, gene clusters, and biosynthesis of trichothecenes and fumonisins in Fusarium. Toxin Rev 2009, 28:198-215.

54. Martin-Hernandez AM, Dufresne M, Hugouvieux V, Melton R, Osbourn A: Effects of targeted replacement of the tomatinase gene on the interaction of septoria lycopersici with tomato plants. MPMI 2000, 13(12):1301-1311.

55. Idnurm A, Howlett BJ: Pathogenicity genes of phytopathogenic fungi. Mol Plant Pathol 2001, 2:241-255.

56. Pareja-Jaime $Y$, Roncero Ml, Ruiz-Roldán MC: Tomatinase from Fusarium oxysporum f. $\mathrm{sp}$. lycopersici is required for full virulence on tomato plants. Mol Plant Microbe Interact 2008, 21(6):728-736.

57. Haas H, Eisendle M, Turgeon BG: Siderophores in Fungal Physiology and Virulence. Ann Rev Phytopathol 2008, 46:149-187.

58. Urade R: Cellular response to unfolded proteins in the endoplasmic reticulum of plants. FEBS Journal 2007, 274:1152-1171.

59. Michielse CB, van Wijk R, Reijnen L, Cornelissen BJ, Rep M: Insight into the molecular requirements for pathogenicity of Fusarium oxysporum f. sp. lycopersici through large-scale insertional mutagenesis. Gen Biol 2009, 10:R4.

60. Chen H, McCaig BC, Melotto M, He SY, Howe GA: Regulation of plant arginase by wounding, jasmonate, and the phytotoxin coronatine. $J$ Biol Chem 2004, 279(44):45998-46007.

61. Koide T, Zaini PA, Moreira LM, Vêncio RZN, Matsukuma AY, Durham AM, Teixeira DC, El-Dorry H, Monteiro PB, da Silva ACR, Verjovski-Almeida S, da Silva AM, Gomes SL: DNA microarray-based genome comparison of a pathogenic and a nonpathogenic strain of Xylella fastidiosa delineates genes important for bacterial virulence. J Bacterio/ 2004, 186(16):5442-5449.

62. Gobert AP, McGee DJ, Akhtar M, Mendz GL, Newton JC, Cheng Y, Mobley HL, Wilson KT: Helicobacter pylori arginase inhibits nitric oxide production by eukaryotic cells: a strategy for bacterial survival. Proc Natl Acad Sci 2001, 98(24):13844-13849.

63. Ramonell KM, Zhang B, Ewing RM, Chen Y, Xu D, Stacey G, Somerville S: Microarray analysis of chitin elicitation in Arabidopsis thaliana. Mol Pl Pathol 2002, 3(5):301-311.

64. Wan J, Dunning MF, Bent AF: Probing plant-pathogen interactions and downstream defense signaling using DNA microarrays. J Func Integr Gen 2002, 2(6):259-273.

65. Schaller A, Stintzi A: Jasmonates in Stress Responses and Development Phytochemistry 2009, 70(13-14):1532-1538.

66. Montalbini P: Xanthine dehydrogenase from leaves of leguminous plants: Purification, characterization and properties of the enzyme. J Plant Physiol 2000, 156(1):3-16.

67. Yesbergenova Z, Yang G, Oron E, Soffer D, Fluhr R, Sagi M: The plant Mohydroxylases aldehyde oxidase and xanthine dehydrogenase have distinct reactive oxygen species signatures and are induced by drought and abscisic acid. Plant J 2005, 42(6):862-76. 
68. Kazan K, Manners JM: Linking development to defense: auxin in plantpathogen interactions. Trends in Plant Science 2009, 14(7):373-382.

69. Luan S, Kudla J, Rodriguez-Concepcion M, Yalovsky S, Gruissem W: Calmodulins and calcineurin B-like proteins: calcium sensors for specific signal response coupling in plants. Plant Cell 2002, 14:S389-S400.

70. Century KS, Holub EB, Staskawicz BJ: NDR1, a locus of Arabidopsis thaliana that is required for disease resistance to both a bacterial and a fungal pathogen. PNAS 1995, 92(14):6597-6601.

71. Martin GB, Bogdanove AJ, Sessa G: Understanding the functions of plant disease resistance proteins. Ann Rev PI Biol 2003, 54:23-61.

72. Joobeur T, King JJ, Nolin SJ, Thomas CE, Dean RA: The Fusarium wilt resistance locus Fom-2 of melon contains a single resistance gene with complex features. Plant J 2004, 39(3):283-297.

73. Rocha PSCF, Sheikh M, Melchiorre R, Fagard M, Boutet S, Loach R, Moffatt B, Wagner $\mathrm{C}$, Vaucheret $\mathrm{H}$, Furner I: The Arabidopsis HOMOLOGY-DEPENDENT GENE SILENCING1 Gene codes for an s-adenosyl-L-homocysteine hydrolase required for DNA methylation-dependent gene silencing. Plant Cell 2005, 17:404-417.

74. Ellendorff U, Fradin EF, de Jonge R, Thomma BPHJ: RNA silencing is required for Arabidopsis defence against Verticillium wilt disease. J Exp Bot 2009, 60:591-602.

75. Padmanabhan C, Zhang $X$, Jin H: Host small RNAs are big contributors to plant innate immunity. Curr Op PI Biol 2009, 12:465-472.

76. Park S-J, Huang Y, Ayoubi P: Identification of expression profiles of sorghum genes in response to green bug phloem feeding using CDNA subtraction and microarray analysis. Planta 2005, 223:932-947.

77. Talboys PW: Resistance to vascular wilt fungi. A Discussion on Disease Resistance in Plants. Proceedings of the Royal Society of London. Series B, Biological Sciences 1972, 181:319-332.

78. Lasserre E, Godard F, Bouquin T, Hernandez J, Pech J-C, Roby D, Balagué C: Differential activation of two ACC oxidase gene promoters from melon during plant development and in response to pathogen attack. Mol Gen Genet 1997, 256:211-222.

79. Belisario A, Luongo L, Corazza L, Gordon TR: Indagini su popolazioni di Fusarium oxysporum f. sp. melonis in Italia. Colture Protette 2000, 3:87-89.

80. Edgington ES, Onghena P: Randomization tests. Chapman and Hall/CRC Press, Boca Raton, Florida;" Fourth 2007.

81. Anderson MJ, Ter Braak C: Permutation tests for multi-factorial analysis of variance. Journal of Statistical Computations and Simulations 2003, 73:85-113.

82. Vos P, Hogers R, Bleeker M, Reijans M, van de Lee $T$, Hornes M, Frijters A, Pot J, Peleman J, Kuiper M, et al: AFLP: a new technique for DNA fingerprinting. Nucleic Acids Res 1995, 23:4407-4414.

83. Bachem CW, van der Hoeven RS, de Bruijn SM, Vreugdenhil D, Zabeau M, Visser RG: Visualization of differential gene expression using a novel method of RNA fingerprinting based on AFLP: analysis of gene expression during potato tuber development. Plant J 1996, 9:745-753.

84. Breyne P, Dreesen R, Cannoot B, Rombaut D, Vandepoele K, Rombauts S, Vanderhaeghen $R$, Inzé $D$, Zabeau M: Quantitative CDNA-AFLP analysis for genome-wide expression studies. Mol Genet Genomics 2003, 269:173-179.

85. Altschul SF, Madden TL, Schaffer AA, Zhang J, Zhang Z, Miller W, Lipman DJ: Gapped BLAST and PSI-BLAST: a new generation of protein database search programs. Nucleic Acids Res 1997, 25:3389-3402.

86. Reid KE, Olsson N, Schlosser J, Peng F, Lund ST: An optimized grapevine RNA isolation procedure and statistical determination of reference genes for real-time RT-PCR during berry development. BMC Plant Biol 2006, 6:27.

doi:10.1186/1471-2164-12-122

Cite this article as: Sestili et al.: Distinct colonization patterns and cDNAAFLP transcriptome profiles in compatible and incompatible interactions between melon and different races of Fusarium oxysporum f. sp. melonis. BMC Genomics 2011 12:122.

\section{Submit your next manuscript to BioMed Central and take full advantage of:}

- Convenient online submission

- Thorough peer review

- No space constraints or color figure charges

- Immediate publication on acceptance

- Inclusion in PubMed, CAS, Scopus and Google Scholar

- Research which is freely available for redistribution

Submit your manuscript at www.biomedcentral.com/submit
Ciomed Central 